Research Article

\title{
Study on Thermally Induced Crack Propagation Behavior of Functionally Graded Materials Using a Modified Peridynamic Model
}

\author{
Fei Wang $\mathbb{D}^{D}$, Yu'e Ma ${ }^{\mathbb{D}}$, Yanning Guo, and Wei Huang \\ School of Aeronautics, Northwestern Polytechnical University, Xi'an 710072, China \\ Correspondence should be addressed to Yu'e Ma; ma.yu.e@nwpu.edu.cn
}

Received 11 August 2019; Accepted 6 November 2019; Published 25 January 2020

Academic Editor: Charles C. Sorrell

Copyright (c) 2020 Fei Wang et al. This is an open access article distributed under the Creative Commons Attribution License, which permits unrestricted use, distribution, and reproduction in any medium, provided the original work is properly cited.

\begin{abstract}
Peridynamic (PD) theory is used to study the thermally induced cracking behavior of functionally graded materials (FGMs). A modified thermomechanical peridynamic model is developed. The thermal crack propagation of a ceramic slab in quenching is calculated to validate the modified PD model. The results predicted by the modified PD model agree with previously published numerical and experimental ones. Compared with the original PD model, the calculation accuracy of the modified PD model for thermal cracking is improved. The thermal cracking in FGMs is also simulated. The effects of material shape, initial temperature, and ceramic fracture toughness on thermal crack propagation behaviors are studied. It can be found that the thermal cracks in FGMs are still in periodical and hierarchical forms. The metal materials in FGMs can prevent crack initiation and arrest the long cracks. The crack number tends to be increased with the increasing initial temperature, while the strengthened ceramic fracture toughness can decrease it.
\end{abstract}

\section{Introduction}

Metals and traditional layered composite materials process high strength and fracture toughness at room temperature. But they become incapable for application at high temperatures. Because of the low fracture toughness of ceramic materials, its employment in heat shielding structure is limited [1]. Functionally graded materials (FGMs) are a new generation of advanced composite materials which combine the advantages of ceramic and metal materials. Because mechanical properties of FGMs continuously change with spatial location, delamination which induces traditional composites or jointed dissimilar materials to fail does not happen in it. FGMs can also provide excellent heat shielding capability and high composite bonding strength with engineered material gradient and orientation [2]. This feature makes FGMs preferably be used in high temperature environments [3]. The applications of FGMs were summarized in [4-6]. Since FGMs are increasingly applied in aerospace structures and components, study on thermally induced crack propagation behavior of FGMs is necessary for assessing and enhancing structural integrity.

Various analytical and computational methods have been studied to simulate thermal fracture of FGMs. The analytical methods such as elastic fracture theoretical method, singular integral method, and homogenous multilayered method (HM method) were mainly employed to derive crack-tip fields and cracking angle. The material properties of FGMs were usually specified as simple functions of spatial locations, for example, the exponential function [7] or the power-law function [8]. The crack-tip thermal stress field and the plastic singular field of FGMs were conducted based on the fracture theory [9]. The Fourier transform can be used to reduce the thermal and mechanical problems of FGMs into integral equation systems [10]. The thermal stress intensity factor (TSIF) in FGMs with a crack was obtained by solving integral equation systems [11]. Petrova and Schmauder [12, 13] employed the integral equation method to evaluate crack deflection angle and TSIF of functionally graded/homogeneous bimaterials with an 
interface crack. In the HM method, FGMs is divided into several layers with constant properties along the graded direction. Guo et al. [14] used the HM method to investigate TSIFs of a piecewise exponential FGM plate with different properties. The explicit monitoring of crack initiation and propagation in FGMs is still difficult to be simulated due to the limitation of analytical techniques.

In order to model crack initiation and propagation in FGMs with complex material properties and loading, some numerical methods such as finite element method (FEM) and extend finite element method (XFEM) were often applied. Fujimoto and Noda [15] used FEM to calculate thermal fracture of the FGM plate with an edge crack and studied the effects of thermal condition and ceramic fracture toughness on crack path. In [16], the crack paths of FGM plate with two cracks were studied by FEM. The remeshing algorithm is needed in FEM to be implemented at every crack increasement [17]. The time-consuming is increased by this algorithm in numerical simulation. Moreover, a fracture criterion such as the maximum tangential stress criterion is required to predict crack kinking angle for every step [18]. The XFEM was implemented to predict thermal crack paths of isotropic and orthotropic FGMs in [19]. Ivanov et al. [20] used XFEM to calculate crack propagation of FGM strips with an edge crack under thermal shock. Pathak [21] applied XFEM to study crack interaction in FGMs under thermal and mechanical loads. The crack discontinuity modelling was facilitated by extrinsically enriched approximation. Bhardwaj et al. [22] used extended isogeometric analysis (XIGA) to simulate cracked FGM plates. In XIGA, XFEM and isogeometric analysis (IGA) were coupled. Nonuniform rational B-splines (NURBS) was used to define geometry and solution. Although XFEM permits crack to propagate through elements without remeshing, some extra crack modelling techniques such as crack growth criterion, level set method, and enrichment functions are required during analysing. Furthermore, coupling of the mesh-free method with FEM is also a choice to calculate crack propagation as well. Pathak [23] performed a method which coupled FEM and extrinsically enriched elementfree Galerkin (XEFG) to analyse crack growth in FGMs under cyclic thermal and mechanical loading environments. The enrichment functions were still required to model crack discontinuity.

To avoid the disadvantages of numerical methods based on continuum mechanics, a nonlocal mechanical framework called peridynamics was proposed by Silling [24]. The basic control equation of peridynamics is reformulated by integration of forces instead of using divergence of stresses. Each material point in peridynamics is connected to others inside a radius region which is called horizon. The interactions between points can be assumed as peridynamic bonds. Discontinuities such as cracks can be introduced by eliminating all the bonds that pass though the crack surface. A bond breaks when its state variables reach a critical value for failure. Damage of a point is characterized by the broken bond number [25]. Then, crack path can be represented by damages of all the points on the structure domain. The extra fracture criterion which determines crack growth direction and extension increment is not required. Other discontinuity modelling technique to overcome mathematical inconsistences is not needed in peridynamics as well. Cracks will naturally propagate when peridynamic bonds break. Additionally, peridynamics also has advantages of the meshless method, owing to the feature of an integrodifferential equation.

Peridynamics has been used to study thermal crack propagation behaviors of brittle solids. Kilic and Madenci [26] employed the bond-based peridynamics (BB-PD) to predict crack growth patterns in quenched glass plates. The simulation results agreed with the experimental observations in [27]. D'Antuono and Morandini [28] gave a weakly coupled thermomechanical ordinary state-based peridynamic (OSB-PD) model and simulated the thermal shock cracking behaviors of a brittle slab and a honeycomb plate. Oterkus and Madenci [29] calculated the crack initiation and propagation of fuel pellet using oxygen diffusion and thermomechanical model within the framework of BB-PD. Zhang and Qiao [30] presented an extended OSB-PD model to predict damage growth of bimaterial structures. Wang et al. [31] developed a coupled BB-PD thermomechanical model to study thermal cracking behavior of ceramic nuclear pellet under power cycles. They also developed a BB-PD model enriched with shear deformation to investigate thermal shock crack branching instability in brittle solids [32]. Furthermore, Wang et al. [33] presented a dual-horizon BB-PD model to simulate thermal fracture. Wang and Zhou [34] introduced the shear failure into OSB-PD and calculated the thermal cracking in rocks. Giannakeas et al. [35] combined the BB-PD and FEM to calculate thermal cracking in ceramics and studied the effects of hot and cold shock in brittle solids. However, numerical studies on thermal crack propagation behavior of FGMs using the peridynamic model have not been published.

In this paper, a modified BB-PD thermomechanical model is developed to calculate thermally induced cracking in FGMs. The peridynamic material parameters in discretized form and the energy weight functions of bond constants are given. The goals of this work are to correct the calculation accuracy of thermal crack propagation and to study the effects of material shape, initial temperature, and ceramic fracture toughness on crack path in FGMs under thermal shock.

\section{Peridynamic Theory}

2.1. Modified Heat Conduction Formulas. The difference between peridynamics and classical local theory is that the peridynamics takes all the material point interactions within a horizon into accounts. However, the classical local theory considers the interactions that are only adjacent to a point. The horizon means a neighborhood of point $\mathbf{x}$ with a limited radius $\delta$ (see Figure 1).

The heat conduction equation based on peridynamics for homogenous materials can be derived by the Euler-Lagrange equation or energy conservation law [36]:

$$
\rho c_{v} \dot{T}(\mathbf{x}, t)=\int_{H_{x}} \mu f_{q}\left(\mathbf{x}^{\prime}, \mathbf{x}, t\right) \mathrm{d} V_{x^{\prime}}+h_{s}(\mathbf{x}, t),
$$




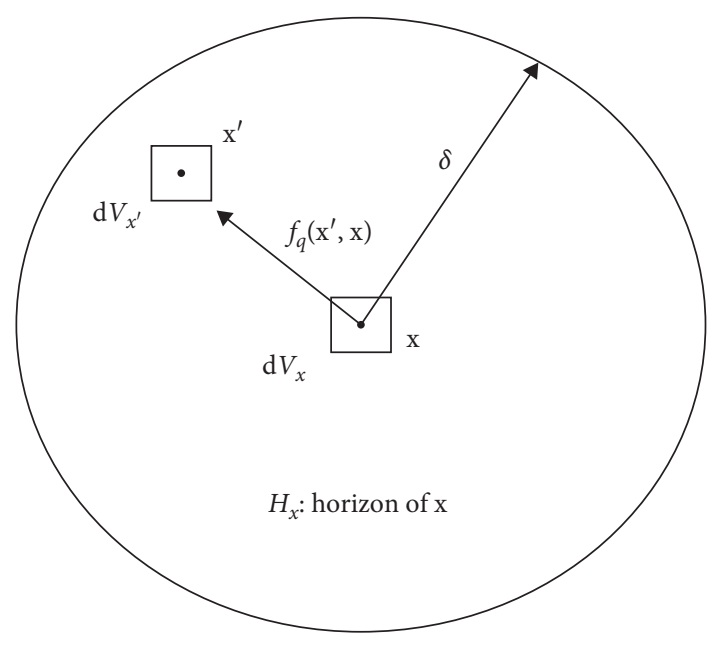

Figure 1: Thermal response function.

where $\rho$ and $c_{v}$ are, respectively, the density and the heat specific of materials. $h_{s}(\mathbf{x}, t)$ is the heat source function per unit volume at point $\mathbf{x} . \mu$ is defined as 0 or $1 . f_{q}\left(\mathbf{x}^{\prime}, \mathbf{x}, t\right)$ is called the thermal response function or the "kernel" function (see Figure 1). The form of $f_{q}\left(\mathbf{x}^{\prime}, \mathbf{x}, t\right)$ for bond-based peridynamics is given as follows [37]:

$$
f_{q}\left(\mathbf{x}^{\prime}, \mathbf{x}, t\right)=\kappa \frac{\tau\left(\mathbf{x}^{\prime}, \mathbf{x}, t\right)}{|\xi|^{n}},
$$

where $\kappa$ is the microconductivity of materials and it means the thermal conductivity in peridynamics. $\xi$ is the distance vector of $\mathbf{x}^{\prime}$ and $\mathbf{x}$; i.e., $\xi=\mathbf{x}^{\prime}-\mathbf{x} . \tau\left(\mathbf{x}^{\prime}, \mathbf{x}, t\right)$ is the temperature difference of $\mathbf{x}^{\prime}$ and $\mathbf{x}$ at time $t$ :

$$
\tau\left(\mathbf{x}^{\prime}, \mathbf{x}, t\right)=T\left(\mathbf{x}^{\prime}, t\right)-T(\mathbf{x}, t) .
$$

The integer $n$ is called the "shape factor." It equals to 0,1 , or 2. More details of "shape factor" can be found in [38].

The classical thermal potential can be given by

$$
Z^{\mathrm{CM}}(\mathbf{x}, t)=\frac{1}{2} \nabla T(\mathbf{x}, t) \cdot k \nabla T(\mathbf{x}, t),
$$

where $\nabla$ is the gradient operator and $k$ is the thermal conductivity in classical heat transfer theory. The peridynamic thermal potential $Z^{\mathrm{PD}}\left(\mathbf{x}^{\prime}, \mathbf{x}, t\right)$ is a function of microthermal potential $z\left(\mathbf{x}^{\prime}, \mathbf{x}, t\right)$ which is given by

$$
Z^{\mathrm{PD}}(\mathbf{x}, t)=\frac{1}{2} \int_{H_{x}} z\left(\mathbf{x}^{\prime}, \mathbf{x}, t\right) \mathrm{d} V_{x^{\prime}}
$$
by

The thermal response function can be related to $z\left(\mathbf{x}^{\prime}, \mathbf{x}, t\right)$

$$
f_{q}=\frac{\partial z}{\partial \tau}
$$

Hence, the microthermal potential can be derived as

$$
z=\frac{\tau^{2}}{2|\xi|^{n}}
$$

When a linear temperature field is applied, $\kappa$ can be obtained from the equivalent relation of classical and peridynamic thermal potential. After the integrals are solved, the expression of $\kappa$ for $2 \mathrm{D}$ in the original peridynamic model are given as

$$
\kappa\left(\mathbf{x}^{\prime}, \mathbf{x}\right)=\frac{2(4-n) k}{h \pi \delta^{4-n}}, \text { for } 2 \mathrm{D},
$$

where $h$ is the thickness.

The microconductivity is obtained under the assumption that material points are always within a full horizon. The surface corrections are needed for the material points located close to surfaces. The surface correction methods were summarized in [39]. In these methods, the correction factors are needed to be derived for revising the full horizon material parameters. Here, a modified peridynamic model is developed to correct surface effect without calculating extra correction factors. The linear temperature field assumption is applied as well. The equivalent relation of classical and peridynamic thermal potential can be also written in discretized form:

$$
k=\frac{\kappa}{4} \sum_{j=1}^{l} \frac{V_{x^{\prime}}}{|\xi|^{n-2}} \text {, for } 2 \mathrm{D} \text {, }
$$

where $l$ is the material point number within a horizon. Thus, the expression of $\kappa$ in the modified peridynamic model can be derived as

$$
\kappa=\frac{4 k}{\sum_{j=1}^{l} V_{x^{\prime}} /|\xi|^{n-2}} \text {, for 2D. }
$$

2.2. Modified Thermomechanical Formulas. The derivation of the peridynamic equation is contained in [40]. The basic motion equation is given by

$$
\rho(\mathbf{x}) \ddot{u}(\mathbf{x}, t)=\int_{H_{x}} \mu \mathbf{f}\left(\mathbf{x}^{\prime}, \mathbf{x}, t\right) \mathrm{d} V_{x^{\prime}}+\mathbf{b}(\mathbf{x}, t),
$$

where $\mathbf{f}$ is the pairwise bond force density function, as shown in Figure 2. The value of this function is decided by material properties and its deformation through the constitutive model. $\ddot{u}$ is the acceleration of point $\mathbf{x}$. The function $\mathbf{b}$ is the body force density.

The pairwise force function $\mathbf{f}$ can be derived from the micropotential $w\left(\mathbf{x}^{\prime}, \mathbf{x}, t\right)$ :

$$
\mathbf{f}\left(\mathbf{x}^{\prime}, \mathbf{x}, t\right)=\frac{\partial w}{\partial \mathbf{\eta}}
$$

in which

$$
\mathbf{\eta}=\mathbf{u}\left(\mathbf{x}^{\prime}, t\right)-\mathbf{u}(\mathbf{x}, t)
$$

and $\mathbf{u}(\mathbf{x}, t)$ is the displacement of $\mathbf{x}$ at time of $t . \mathrm{A}$ micropotential that leads to a linear thermomechanical material model can be expressed as [41]

$$
w=\frac{1}{2} c s^{2}|\xi|-c \alpha \Delta T s|\xi|,
$$

where $c$ is a parameter called the micromodulus and $\alpha$ is the coefficient of thermal expansion. $\Delta T$ is temperature 


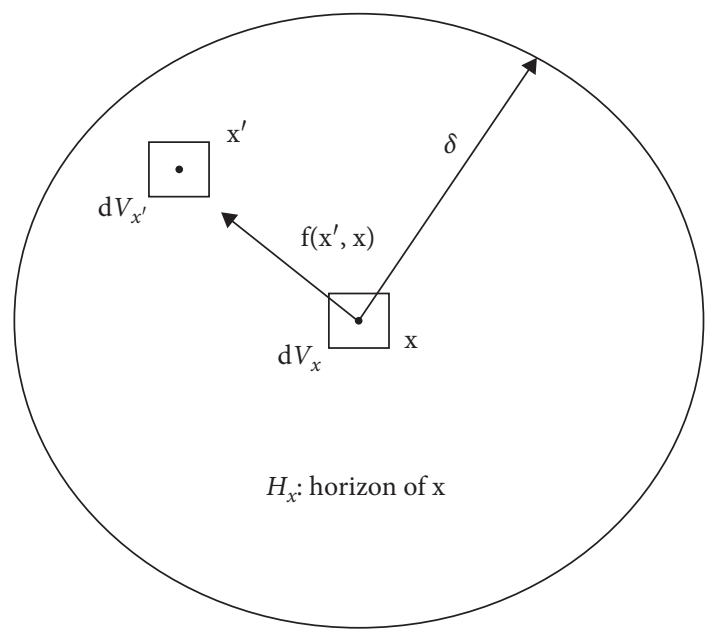

Figure 2: Pairwise bond force density function.

difference of a bond. $\Delta T=\left[T\left(\mathbf{x}^{\prime}, t\right)+T(\mathbf{x}, t)-T_{0}\left(\mathbf{x}^{\prime}, t\right)-T_{0}\right.$ $\left.\left(\mathbf{x}^{\prime}, t\right)\right] / 2 . T_{0}$ is initial temperature field. $s$ is the bond strain, and it is defined by

$$
s=\frac{|\eta+\xi|-|\xi|}{|\xi|} .
$$

Corresponding to the micropotential given in equation (14), the pairwise force density function can be given as

$$
\mathbf{f}\left(\mathbf{x}^{\prime}, \mathbf{x}, t\right)= \begin{cases}c(s-\alpha \Delta T) \mathbf{M}, & \text { if }|\xi|<\delta, \\ 0, & \text { ortherwise }\end{cases}
$$

where $\mathbf{M}$ is a unit vector in the direction of deformed bond. It can be written as

$$
M=\frac{\eta+\xi}{|\eta+\xi|}
$$

The peridynamic strain energy density can be given by

$$
W^{\mathrm{PD}}(\mathbf{x}, t)=\frac{1}{2} \int_{H_{x}} w\left(\mathbf{x}^{\prime}, \mathbf{x}, t\right) \mathrm{d} V_{x^{\prime}} .
$$

The parameter micromodulus $c$ can be derived by matching the peridynamic strain energy density and the value from the classical elasticity theory. For plane stress, the micromodulus in the original peridynamic model can be derived as

$$
c=\frac{12 K}{h \pi \delta^{3}}
$$

where $K$ is the bulk modulus, and it can be given as

$$
K=\frac{E}{2(1-v)}
$$

where $E$ is the elastic modulus and $\nu$ is Poisson's ratio.

Similarly, the micromodulus in the modified peridynamic model can be derived as

$$
c=\frac{8 K}{\sum_{j=1}^{l}|\xi| V_{x^{\prime}}} \text {. }
$$

The thermomechanical damage can be introduced by the scalar-valued function $\mu$ which is defined by

$$
\mu\left(\mathbf{x}^{\prime}, \mathbf{x}, t\right)= \begin{cases}1, & s-\alpha \Delta T<s_{0}, \\ 0, & \text { ortherwise }\end{cases}
$$

where $s_{0}$ is the critical relative elongation for bond failure. Silling and Askari [25] related $s_{0}$ with the fracture energy $G_{\text {IC }}$ for plane stress, and it can be derived as

$$
s_{0}=\sqrt{\frac{4 \pi G_{\mathrm{IC}}}{9 E \delta}} .
$$

The local damage at a point can be directly defined as the ratio of broken bond number to total initial bond number:

$$
\varphi(\mathbf{x}, t)=1-\frac{\int_{H_{x}} \mu\left(\mathbf{x}^{\prime}, \mathbf{x}, t\right) \mathrm{d} V_{x^{\prime}}}{\int_{H_{x}} \mathrm{~d} V_{x^{\prime}}} .
$$

2.3. Microconductivity and Micromodulus of a Bond. When material point is located closely to surface or the material is heterogeneous, the material parameters (i.e., microconductivity or micromodulus) of the PD model vary with the point location. Hence, the parameters of a bond are determined by the values on two material points of this bond. Cheng et al. [42] applied the average value function to calculate bond constants of heterogeneous materials. In this work, the energy weight functions are used to evaluate bond constants. The microconductivity and micromodulus of a bond can be, respectively, expressed as

$$
\begin{aligned}
& \kappa=G_{t h x} \kappa_{x}+G_{t h x^{\prime}} \kappa_{x^{\prime}}, \\
& c=G_{e x} c_{x}+G_{e x^{\prime}} c_{x^{\prime}},
\end{aligned}
$$

where $G_{t h}$ and $G_{e}$ are the weight parameters of microconductivity and micromodulus. They are, respectively, defined as

$$
\begin{aligned}
G_{t h x} & =\frac{1}{2} h(\mathbf{x}), \\
G_{t h x^{\prime}} & =\frac{1}{2} h\left(\mathbf{x}^{\prime}\right), \\
G_{e x} & =\frac{1}{2}\left(\left[\frac{r_{x}\left(\mathbf{x}^{\prime}, \mathbf{x}\right)}{g_{x}(\mathbf{x})}\right]^{2}+\left[\frac{r_{y}\left(\mathbf{x}^{\prime}, \mathbf{x}\right)}{g_{y}(\mathbf{x})}\right]^{2}\right)^{-1 / 2}, \\
G_{e x^{\prime}} & =\frac{1}{2}\left(\left[\frac{r_{x}\left(\mathbf{x}^{\prime}, \mathbf{x}\right)}{g_{x}\left(\mathbf{x}^{\prime}\right)}\right]^{2}+\left[\frac{r_{y}\left(\mathbf{x}^{\prime}, \mathbf{x}\right)}{g_{y}\left(\mathbf{x}^{\prime}\right)}\right]^{2}\right)^{-1 / 2},
\end{aligned}
$$

where 


$$
\begin{aligned}
h(\mathbf{x}) & =\frac{Z^{\mathrm{CM}}(\mathbf{x})}{Z^{\mathrm{PD}}(\mathbf{x})}, \\
h\left(x^{\prime}\right) & =\frac{Z^{\mathrm{CM}}\left(\mathbf{x}^{\prime}\right)}{Z^{\mathrm{PD}}\left(\mathbf{x}^{\prime}\right)}, \\
\mathbf{g}(\mathbf{x}) & =\left[g_{x}(\mathbf{x}), g_{y}(\mathbf{x})\right]^{T}=\left[\frac{W_{x}^{\mathrm{CM}}(\mathbf{x})}{W_{x}^{\mathrm{PD}}(\mathbf{x})}, \frac{W_{y}^{\mathrm{CM}}(\mathbf{x})}{W_{y}^{\mathrm{PD}}(\mathbf{x})}\right]^{T}, \\
\mathbf{g}\left(\mathbf{x}^{\prime}\right) & =\left[g_{x}\left(\mathbf{x}^{\prime}\right), g_{y}\left(\mathbf{x}^{\prime}\right)\right]^{T}=\left[\frac{W_{x}^{\mathrm{CM}}\left(\mathbf{x}^{\prime}\right)}{W_{x}^{\mathrm{PD}}\left(\mathbf{x}^{\prime}\right)}, \frac{W_{y}^{\mathrm{CM}}\left(\mathbf{x}^{\prime}\right)}{W_{y}^{\mathrm{PD}}\left(\mathbf{x}^{\prime}\right)}\right]^{T}, \\
\mathbf{r} & =\frac{\mathbf{x}^{\prime}-\mathbf{x}}{\left|\mathbf{x}^{\prime}-\mathbf{x}\right|}=\left[r_{x}, r_{y}\right]^{T} \cdot
\end{aligned}
$$

The variables $Z$ and $W$ are thermal potential and strain energy density of a material point. The superscripts CM and PD mean continuum mechanics and peridynamics. The values of $Z$ and $W$ can be calculated by equations (5) and (18) with the linear field assumption. $\mathbf{r}$ is the direction vector of point $\mathbf{x}$ and $\mathbf{x}^{\prime}$.

\section{Numerical Implementations}

The time differential term in heat conduction equation is replaced by forward difference form. The adaptive dynamic relaxation (ADR) technique is chosen to calculate the quasistatic displacements because it is reliable and has a simple algorithm [41, 43]. The Gauss midpoint quadrature scheme is applied to calculate the integrations in heat conduction and motion equations. The volume correction is also considered in both calculations [36]. The peridynamic numerical algorithm for thermally induced cracking is shown in Figure 3.

In Figure $3, t, t_{n}$, and $t_{c}$ are the calculating time of heat conduction, the total calculating time, and the time step for cracking, respectively. int means that the value type is integer. For example, when $t_{c}=1 \mathrm{~s}$, the cracking procedure is implemented for every second.

\section{Validation}

4.1. Ceramic Slab in Quenching. To validate the modified thermomechanical peridynamic model, the $\mathrm{Al}_{2} \mathrm{O}_{3}$ ceramic slab in the quenching test is simulated and thermal crack paths predicted by the modified peridynamic model are compared with the previously published numerical and experimental results. Due to the symmetry of the ceramic slab, only lower left part is calculated. In Figure 4, the length and the width are $25 \mathrm{~mm}$ and $5 \mathrm{~mm}$, respectively. The material properties of $\mathrm{Al}_{2} \mathrm{O}_{3}$ ceramics are shown in Table 1 [33]. The vertical displacement and velocity are fixed on top surface. The horizontal displacement and velocity are fixed on the right surface. Moreover, the adiabatic boundary conditions are assumed on top and right surfaces. The convective heat transfer conditions are applied on bottom and left surfaces [33]:

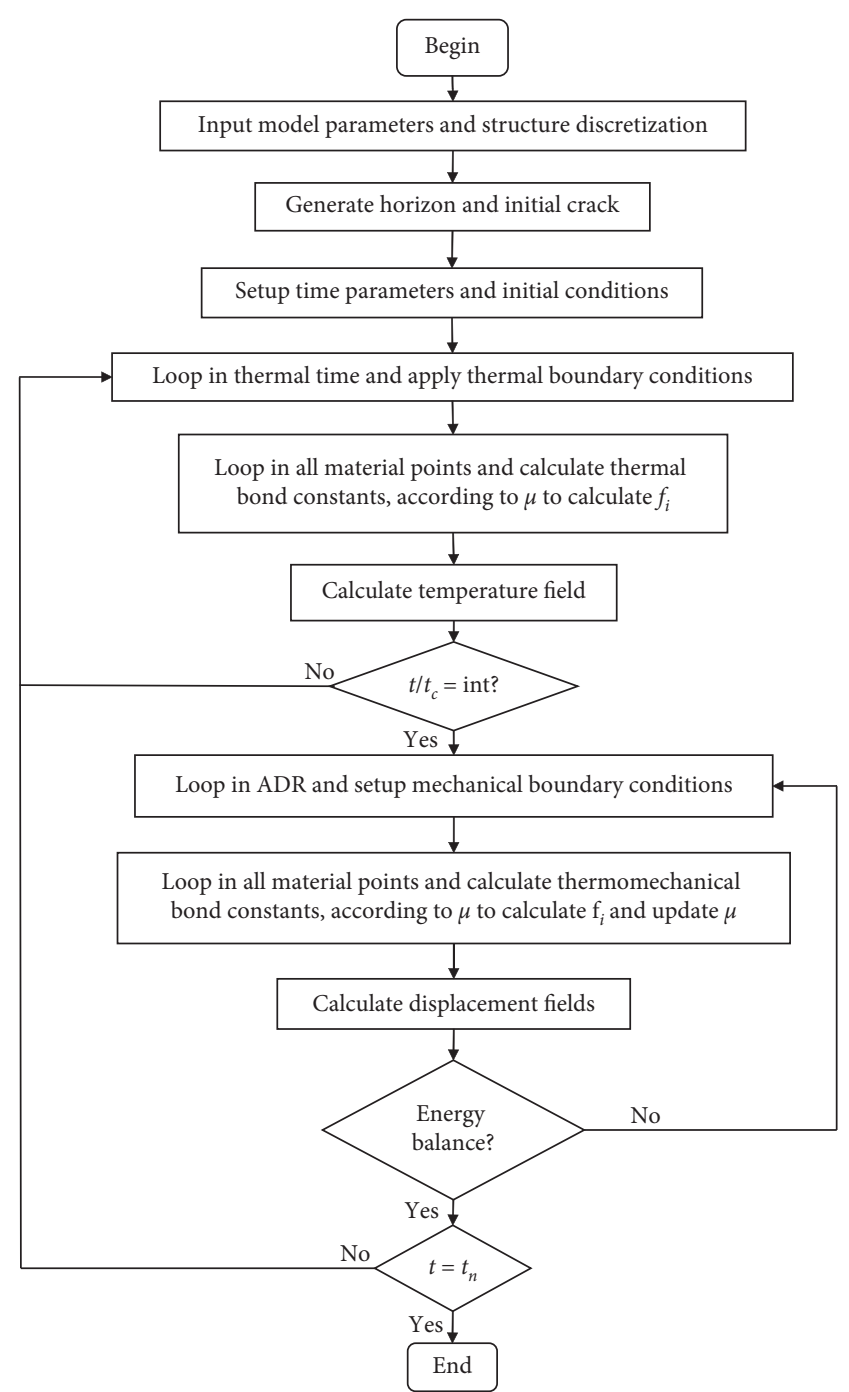

FIgURE 3: Peridynamic numerical algorithm for thermal cracking.

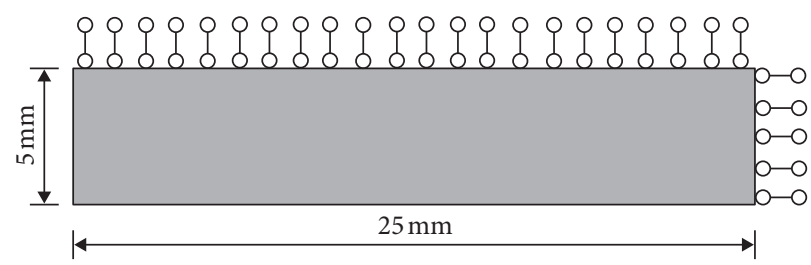

FIgURE 4: $\mathrm{Al}_{2} \mathrm{O}_{3}$ ceramic slab in quenching.

$$
h_{s}(\mathbf{x}, t)=h_{c} \frac{\left(T_{\infty}-T(\mathbf{x}, t)\right)}{\Delta}
$$

where $h_{c}$ is the convective heat transfer coefficient, $T_{\infty}$ is the environment temperature, and $\Delta$ is the cross-sectional area.

The convective heat transfer coefficient $h_{c}$ under different initial temperatures is listed in Table 2. The specified value of $h_{c}$ is dissimilar due to the differences of numerical methods and mesh densities. The time step for heat conduction $\Delta t=1 e-5 \mathrm{~s}$ and the time step for cracking $t_{c}=2 e-3 \mathrm{~s}$ are specified. Plane stress is assumed. 
TABLE 1: Material properties of $\mathrm{Al}_{2} \mathrm{O}_{3}$ ceramics.

\begin{tabular}{lcccccc}
\hline$E(\mathrm{GPa})$ & $v$ & $\rho\left(\mathrm{kg} / \mathrm{m}^{3}\right)$ & $k\left(\mathrm{~J} /\left(\mathrm{m} \cdot{ }^{\circ} \mathrm{C}\right)\right)$ & $c_{v}\left(\mathrm{~J} /\left(\mathrm{kg} \cdot{ }^{\circ} \mathrm{C}\right)\right)$ & $\alpha\left(1 /{ }^{\circ} \mathrm{C}\right)$ & $G_{\mathrm{IC}}\left(\mathrm{J} / \mathrm{m}^{2}\right)$ \\
\hline 370 & $1 / 3$ & 3980 & 31 & 880 & $7.5 e-6$ & 24.3 \\
\hline
\end{tabular}

TABLE 2: Convective heat transfer coefficient $h_{c}$ under different initial temperatures $\left(\mathrm{W} / \mathrm{m}^{2}{ }^{\circ} \mathrm{C}\right)$.

\begin{tabular}{|c|c|c|c|c|c|}
\hline$T_{0}$ & $300^{\circ} \mathrm{C}$ & $350^{\circ} \mathrm{C}$ & $400^{\circ} \mathrm{C}$ & $500^{\circ} \mathrm{C}$ & $600^{\circ} \mathrm{C}$ \\
\hline Present work & 65000 & 85000 & 82000 & 65000 & 45000 \\
\hline Jiang et al. [44] & 54500 & 100000 & 86000 & 57000 & 45000 \\
\hline Li et al. [45] & 65000 & 90000 & 82000 & 70000 & 60000 \\
\hline
\end{tabular}

According to the results of Sicsic et al. [46], the thermal crack initiation is related to thermal shock and geometry as well as the characteristic length of materials. In [47], the characteristic length of $\mathrm{Al}_{2} \mathrm{O}_{3}$ ceramics is specified as $9.2 e-5 \mathrm{~m}$. Silling [48] suggested that the horizon size in peridynamics is equivalent to the material characteristic length. Hence, the point spacing $\Delta x$ is selected as $2.5 e-5 \mathrm{~m}$ and $m=3.015$. The horizon size can be obtained by $\delta=m \Delta x \approx 7.6 e-5 \mathrm{~m}$, which is less than the characteristic length of $\mathrm{Al}_{2} \mathrm{O}_{3}$ ceramics.

4.2. Results and Comparisons. The thermally induced crack propagation in the ceramic slab at an initial temperature of $500^{\circ} \mathrm{C}$ is calculated. The results predicted by the modified PD model in present work are compared with the published numerical ones (see Figure 5). Because the selected time step and $h_{c}$ value are different, the calculation times of crack initiating and crack propagating to the same length have a slight difference.

In Figure 5, the thermally induced cracks are initiated on the fixed free surfaces (bottom and left surfaces) of the ceramic slab where the thermal load is applied (see Figure 5(a)). The initiated directions of the cracks are perpendicular to the edges of the slab, and the thermal cracks are basically parallel to each other. The crack lengths are similar, and initiated locations are equally spaced. Some cracks are arrested over calculation time, and some are driven to propagate toward the inner of the slab (see Figure 5(b) and Figure 5(c)). Hence, the cracks can be classified into different levels according to their lengths. During the crack propagating, the oscillations and slight turning of crack paths can be found. But the cracks are still spaced equally and the crack lengths still keep in hierarchical form (see Figure 5(d)).

In the results predicted by the modified PD model, the surface damages on the skin of the slab are occurred (see Figure 6). The damages are located at the middle parts of the bottom and left surfaces. Crack initiations on these parts are delayed, but this effect on crack propagation result can be neglected. Furthermore, the damages also can be found at the left-lower corner. The reason is that the material is softened by the surface effect of the PD model.

The thermal crack paths at different initial temperatures are calculated. The crack paths predicted by the modified PD model are compared with the experimental ones from [41], as shown in Figure 7. The periodical and hierarchical forms of the thermal cracks can be captured by the modified PD model.

It is assumed that $a$ is the depth of crack tip to the slab edge. $L_{2}$ is the width of the ceramic slab which is equal to $5 \mathrm{~mm}$. Dimensionless crack depth $\bar{a}=a / L_{2}$. The dimensionless depth can be used to classify the thermal cracks into three hierarchical levels. When $\bar{a}>0.6$, the crack is defined as long crack. If $0.3<\bar{a}<0.6$, the thermal crack is defined as medium-sized crack. When $\bar{a}<0.3$, the crack is called as short cracks. The short, medium-sized, long, and total crack numbers are counted from the modified PD simulation results and the experimental tests (see Figure 8). The maximum error number of short cracks predicted by the modified PD model is 3 when $T_{0}=400^{\circ} \mathrm{C}$, while it is 1 for the predicted medium-sized cracks when $T_{0}=400^{\circ} \mathrm{C}$. For the long cracks, it is also 1 at $T_{0}=300^{\circ} \mathrm{C}$, and for total crack number, it is 2 at $T_{0}=300^{\circ} \mathrm{C}$ and $T_{0}=400^{\circ} \mathrm{C}$. So, it can be found that the thermal crack numbers reproduced by the modified PD model are in good agreement with the experimental results.

The thermal crack paths at $T_{0}=600^{\circ} \mathrm{C}$ are also calculated, and they are shown in Figure 9(b). The previous published experimental and numerical results are shown in Figure 9 as well. Compared with the original PD model result, the skin damages on bottom and left surfaces predicted by the modified PD model are thinner. Hence, the surface correction of the modified $\mathrm{PD}$ model is better than that of the original one. The periodical and hierarchical forms of the thermal cracks in the modified PD model result are more precise (see Figures 9(b) and 9(c)).

The crack numbers at $T_{0}=600^{\circ} \mathrm{C}$ are given in Figure 10 . The differences of short, medium-sized, long, and total crack numbers between the modified PD model results and the experimental ones are, respectively, 1, 0, 0, and 1 . The crack number differences for the original PD model are 19, 4, 1, and 13. The crack numbers predicted by the modified PD model agree with the experimental results. Compared with the results of the original PD model, the calculations of the modified model are relatively more satisfying. The crack number differences calculated by FEM are 2, 1, 0, and 2. For the nonlocal approach, they are 15, 1, 1, and 17. The short cracks are initiated more than that observed from the experiment. 

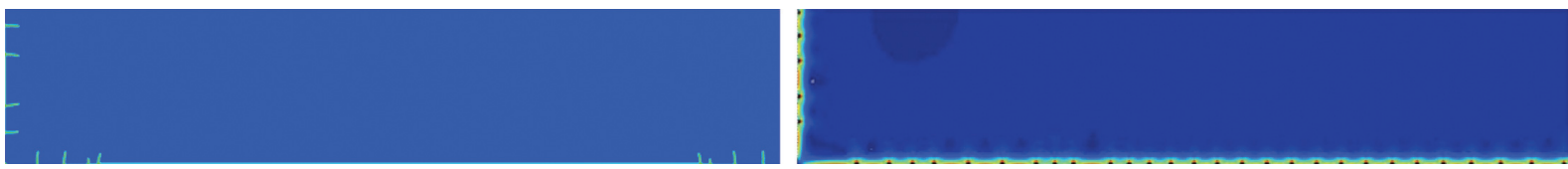

(a)
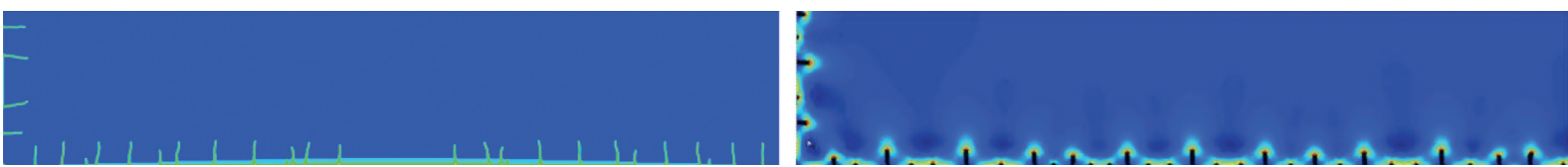

(b)
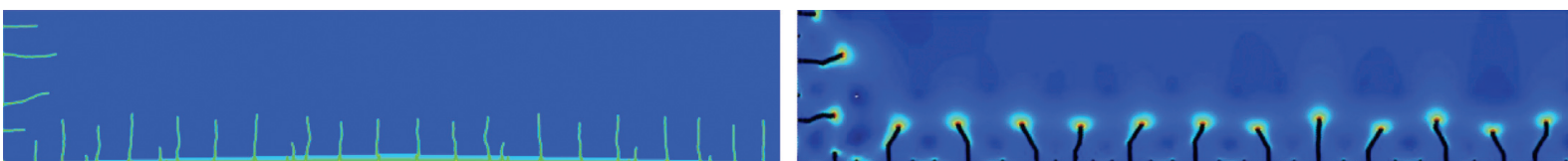

(c)
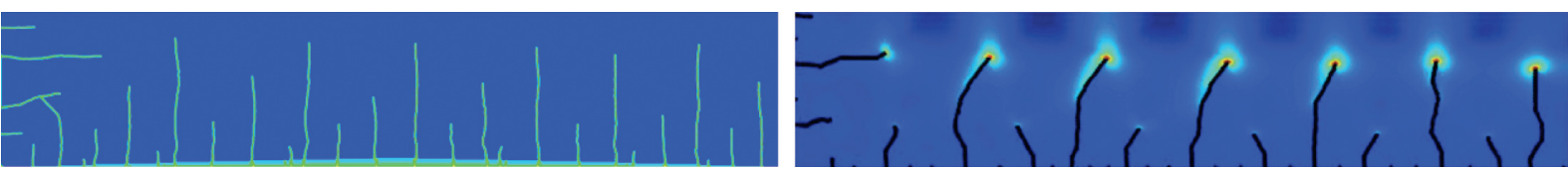

(d)

FIGURE 5: Thermally induced crack initiation and propagation (left side: results predicted by the modified PD model in present work; right side: numerical results in [49] $\left(T_{0}=500^{\circ} \mathrm{C}\right)$ ): (a) $t=0.002 \mathrm{~s}$ and $t=0.0031 \mathrm{~s}$; (b) $t=0.006 \mathrm{~s}$ and $t=0.0075 \mathrm{~s}$; (c) $t=0.05 \mathrm{~s}$ and $t=0.0501 \mathrm{~s}$; (d) $t=0.9 \mathrm{~s}$ and $t=1.1552 \mathrm{~s}$.

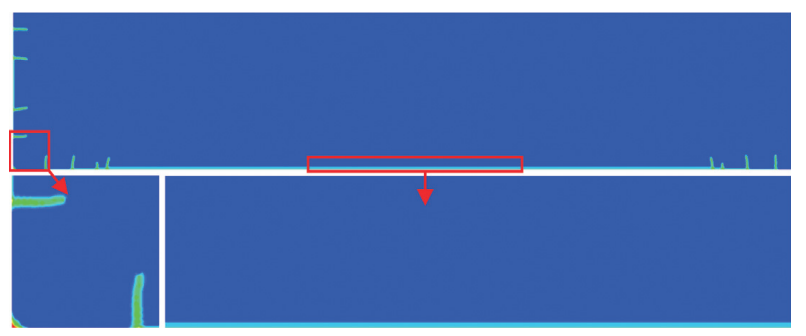

FIGURE 6: Surface damage on slab skin (result of the modified PD model in present work, $t=0.002 \mathrm{~s})$.

\section{Thermal Crack Propagation Behavior in FGMs}

5.1. Sizes and Material Properties of FGM Slab. The FGM slab is composed by $\mathrm{Al}$ metal coating $\mathrm{Al}_{2} \mathrm{O}_{3}$ ceramics (see Figure 11). The geometries and the boundary conditions of the FGM slab are as the same as those in Figure 4.

The FGM slab has a continuously graded composition profile of metal and ceramics. The gradient direction is along the vertical coordinate. The material properties of the FGM slab are assumed as [15]

$$
\begin{aligned}
k & =\frac{k_{0}\left[\left(1-P_{y}^{2 / 3}\right) k_{0}+P_{y}^{2 / 3} k_{a}\right]}{P_{y}^{1 / 3} k_{0}+\left(1-P_{y}^{1 / 3}\right)\left[\left(1-P_{y}^{2 / 3}\right) k_{0}+P_{y}^{2 / 3} k_{a}\right]}, \\
k_{0} & =k_{c}\left[1+\frac{3\left(k_{m}-k_{c}\right) V_{m}}{3 k_{c}+\left(k_{m}-k_{c}\right) V_{c}}\right], \\
\rho & =\rho_{0}\left(1-P_{y}\right)+\rho_{a} P_{y},
\end{aligned}
$$

$$
\begin{aligned}
\rho_{0} & =\rho_{m} V_{m}+\rho_{c} V_{c}, \\
c_{v} & =\frac{c_{0} \rho_{0}\left(1-P_{y}\right)+c_{a} \rho_{a} P_{y}}{\rho_{0}\left(1-P_{y}\right)+\rho_{a} P_{y}}, \\
c_{0} & =\frac{c_{m} \rho_{m} V_{m}+c_{c} \rho_{c} V_{c}}{\rho_{m} V_{m}+\rho_{c} V_{c}}, \\
\nu & =v_{0}, \\
v_{0} & =v_{m} V_{m}+v_{c} V_{c}, \\
E & \left.=\frac{1+P_{y}\left(5+v_{0}\right)\left(37-8 v_{0}\right) / 8\left(1+P_{y}\right)\left(23+8 v_{0}\right)}{1-v^{2 / 3}}\right] \\
E_{0} & =E_{c}\left[\frac{E_{c}+\left(E_{m}-E_{c}\right) V_{m}^{2 / 3}}{E_{c}+\left(E_{m}-E_{c}\right)\left(V_{m}^{2 / 3}-V_{m}\right)}\right] \\
\alpha & =\alpha_{0}, \\
\alpha_{0} & =\frac{\left(1-v_{c}\right) \alpha_{m} E_{m} V_{m}+\left(1-v_{m}\right) \alpha_{c} E_{c} V_{c}}{\left(1-v_{c}\right) E_{m} V_{m}+\left(1-v_{m}\right) E_{c} V_{c}}, \\
K_{\mathrm{IC}} & K_{\mathrm{IC}-m} V_{m}+K_{\mathrm{IC}-c} V_{c},
\end{aligned}
$$

where $k, \rho$, and $c_{v}$ are, respectively, the thermal conductivity, the density, and the specific heat of FGMs. $v, E, \alpha$, and $K_{\mathrm{IC}}$ are Poisson's ratio, the elastic modulus, the linear thermal expansion coefficient, and the fracture toughness. The relation of critical energy release rate $G_{\mathrm{IC}}$ and fracture toughness $K_{\mathrm{IC}}$ for plane stress are given by 

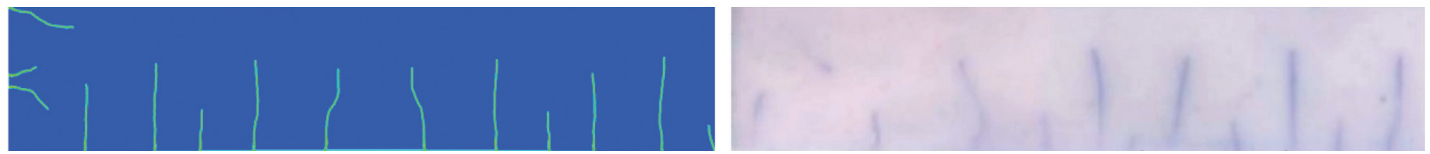

(a)
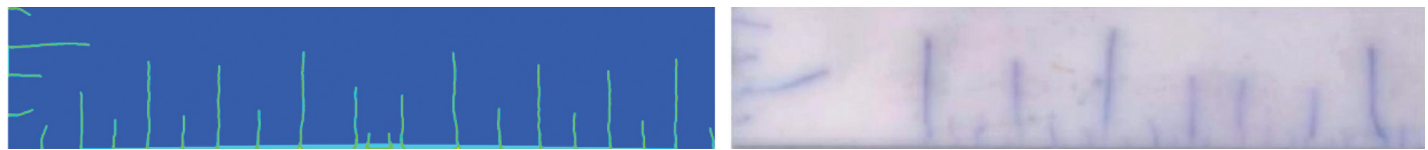

(b)
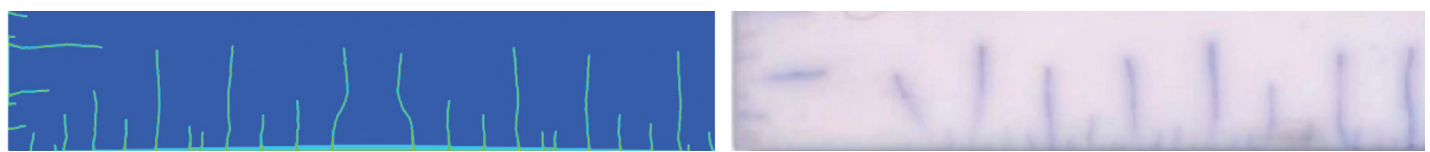

(c)
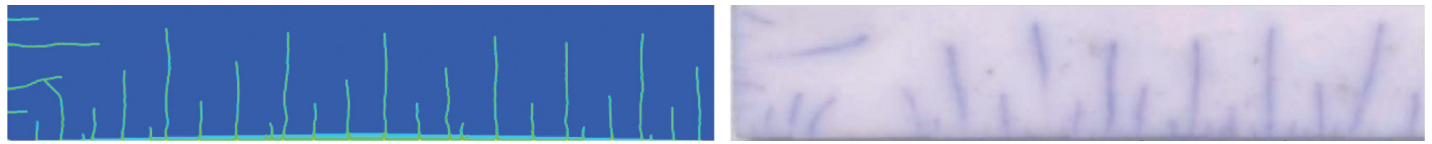

(d)

Figure 7: Thermal crack paths at different initial temperatures (left side: results predicted by the modified PD model in present work; right side: experimental results in [44]): (a) $T_{0}=300^{\circ} \mathrm{C}$; (b) $T_{0}=350^{\circ} \mathrm{C}$; (c) $T_{0}=400^{\circ} \mathrm{C}$; (d) $T_{0}=500^{\circ} \mathrm{C}$.

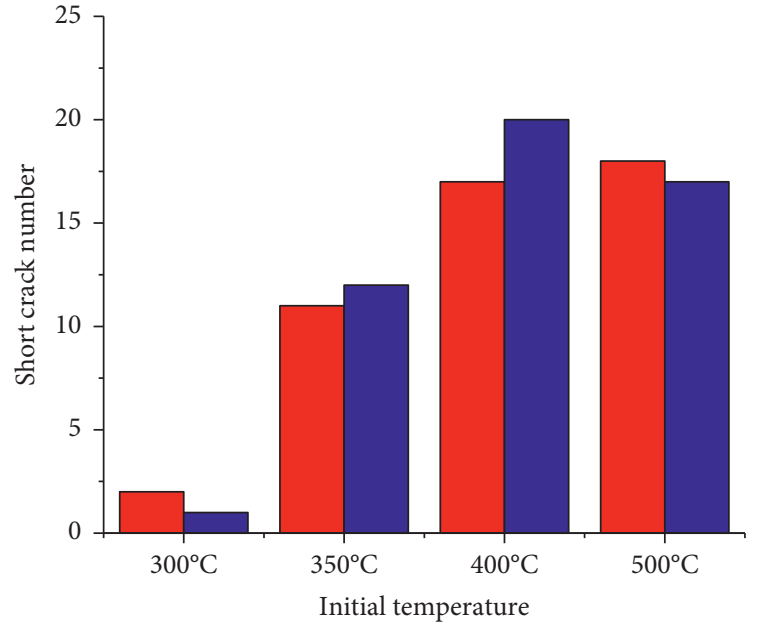

Modified PD

Experiment

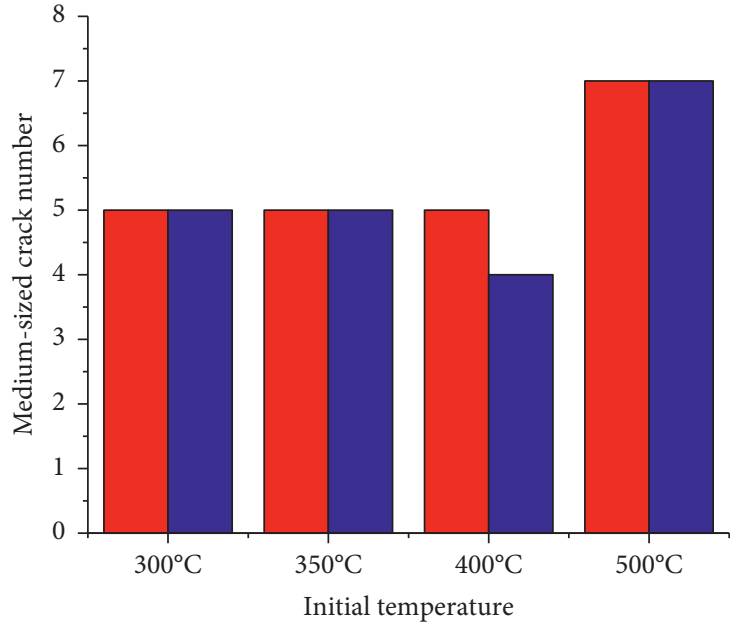

Modified PD

Experiment

(a)

Figure 8: Continued. 


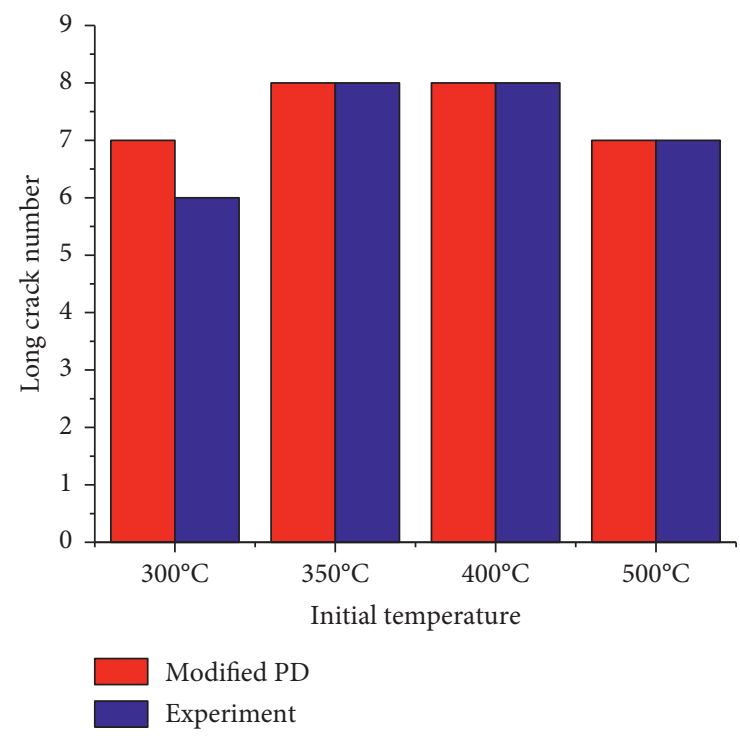

(c)

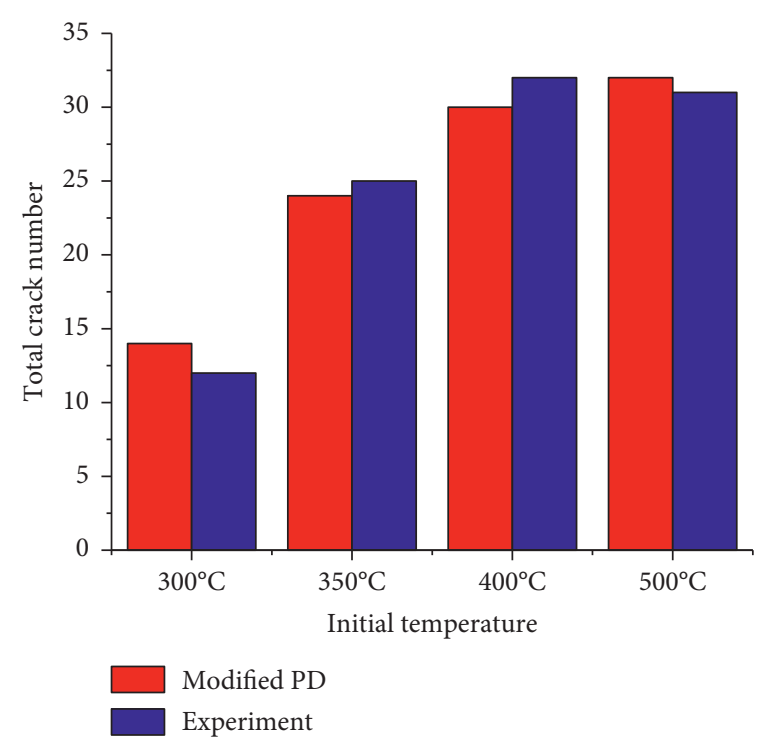

(d)

FIGURE 8: Thermal crack numbers at different initial temperatures: (a) short crack numbers; (b) medium-sized crack numbers; (c) long crack numbers; (d) total crack numbers.

$$
G_{\mathrm{IC}}=\frac{K_{\mathrm{IC}}^{2}}{E}
$$

The subscripts $m, c$, and $a$ indicate $\mathrm{Al}$ metal, $\mathrm{Al}_{2} \mathrm{O}_{3}$ ceramics, and air. The material properties of $\mathrm{Al}_{2} \mathrm{O}_{3}$ are given in Table 1. The properties of $\mathrm{Al}$ and air are shown in Table 3.

Variable $P_{y}$ is the porosity of FGMs along the vertical direction. The volume fractions of metal and ceramics (i.e., $V_{m}$ and $V_{c}$ ) are defined as

$$
\begin{aligned}
V_{m} & =\left(\frac{y}{b}\right)^{M_{y}}, \\
V_{c} & =1-V_{m} .
\end{aligned}
$$

The porosity is expressed as

$$
P_{y}=P_{y}\left(\frac{y}{b}\right)^{n_{y}}\left[1-\left(\frac{y}{b}\right)^{z_{y}}\right]
$$

where

$$
\frac{\left(n_{y}+z_{y}\right) / z_{y}}{\left[1-n_{y} /\left(n_{y}+z_{y}\right)\right]} \geq A_{y} \geq 0
$$

in which $A_{y}, n_{y}$, and $z_{y}$ are the control parameters of FGM porosity. Here, $A_{y}=0$ is specified. The values of $n_{y}$ and $z_{y}$ are selected as 1 . The width of FGM slab $b=5 \mathrm{~mm} . M_{y}$ is the material shape parameter. The volume fraction $V_{m}$ varies with vertical coordinate $y$ and $M_{y}$. It is shown in Figure 12.

5.2. The Effect of Material Shape of FGMs. The temperature fields and thermal crack paths of FGM slab in quenching with different material shape parameters are calculated. The material shape parameter $M_{y}$ is specified as shown in Figure 12. The environment and initial temperatures are $20^{\circ} \mathrm{C}$ and $500^{\circ} \mathrm{C}$. The convective heat transfer coefficient $h_{c}$ is given in Table 2. The temperature fields and the thermally induced crack paths of the FGM slab at $t=1 \mathrm{~s}$ are shown in Figures 13 and 14.

In Figure 13, the material shape parameter has a slight effect on temperature field in this case. Due to the increasing of the material shape parameter, the volume fraction of ceramics is enhanced. Hence, temperature on the lower side of the FGM slab is decreased with the increasing material shape parameter. The temperature jumps are more obvious as a result of thermal crack initiation and propagation.

In Figure 14, there are no thermal crack initiated when the material shape parameter $M_{y}$ is selected as 0.01 and 0.2 (Figures 14(a) and 14(b)). While $M_{y}=1$ (Figure 14(c)), three cracks are initiated on the right-lower side of the FGM slab which are located close to the fixed boundary. At $M_{y}=5$ (Figure 14(d)), many cracks are observed on the lower side of the FGM slab. The thermal cracks are still in periodical and hierarchical forms. But the cracks are arrested at the horizonal center line, and no thermal crack is initiated at the left-upper side. At $M_{y}=70$ (Figure 14(e)), the cracks are not only initiated on the lower side of the FGM slab but also produced on the left side. And their lengths are obviously greater than those when $M_{y}=5$.

In Figure 15, the total crack number is increased when $M_{y}$ is increasing. With the increasing $M_{y}$, the fracture toughness of FGMs is softened. In Table 4, the crack length is increased when $M_{y}$ becomes larger. The existence of metal makes the fracture toughness of FGMs strengthened. It can prevent cracks from initiating and arrest them.

5.3. The Effect of Initial Temperature. The temperature fields and thermal crack paths with different initial temperatures are calculated. The initial temperature $T_{0}$ is, respectively, selected as $300^{\circ} \mathrm{C}, 350^{\circ} \mathrm{C}, 400^{\circ} \mathrm{C}, 500^{\circ} \mathrm{C}$, and $600^{\circ} \mathrm{C}$. 


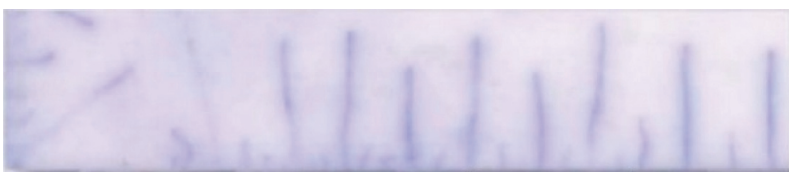

(a)

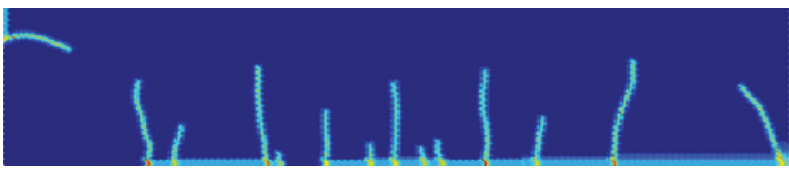

(c)

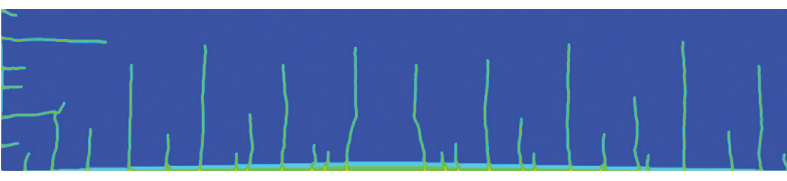

(b)

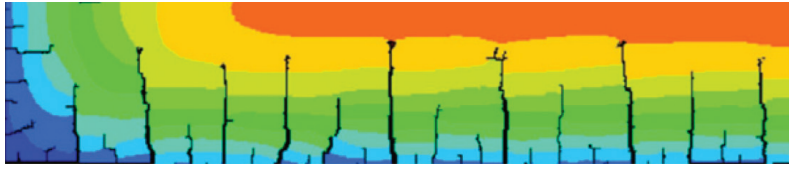

(d)

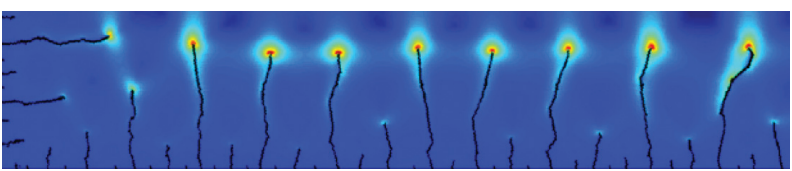

(e)

FIGURE 9: Crack paths predicted by different methods $\left(T_{0}=600^{\circ} \mathrm{C}\right)$ : (a) experimental result [44]; (b) result of the modified PD model in present work; (c) result of the original PD model [31]; (d) result of FEM [50]; (e) result of nonlocal approach [49].

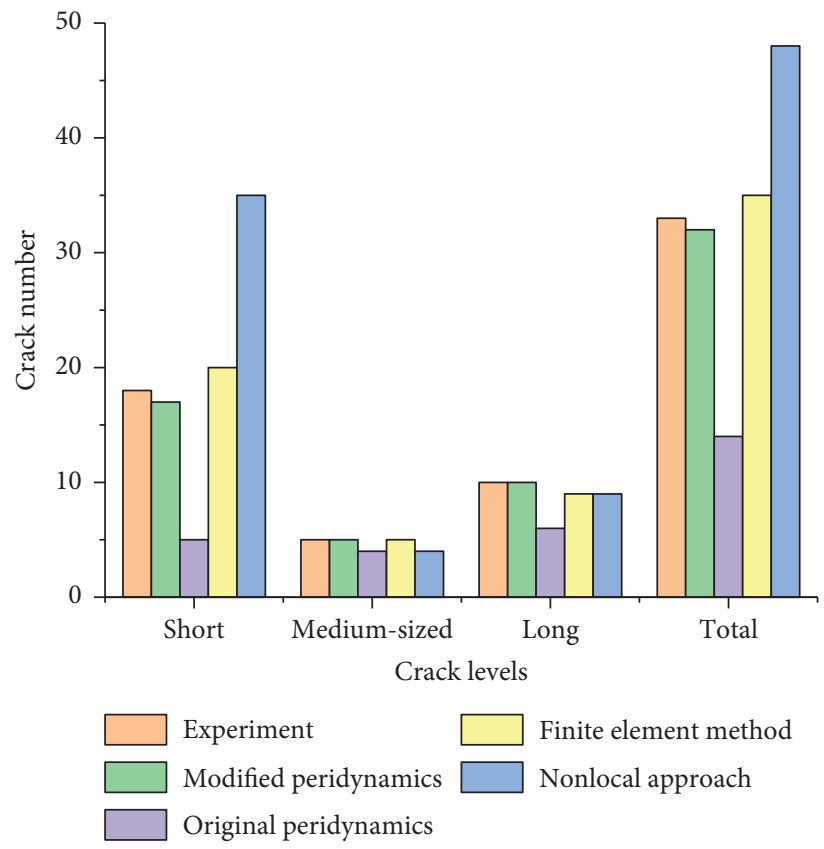

Figure 10: Crack number predicted by different methods $\left(T_{0}=600^{\circ} \mathrm{C}\right)$.

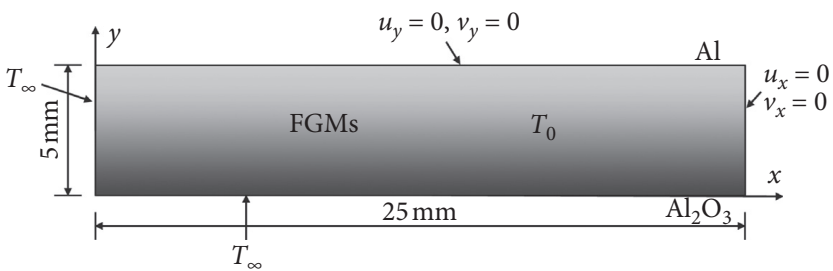

FIGURE 11: FGM slab in quenching. 
TABle 3: Material properties of $\mathrm{Al}$ and air.

\begin{tabular}{lccccccc}
\hline Materials & $E(\mathrm{GPa})$ & $v$ & $\rho\left(\mathrm{kg} / \mathrm{m}^{3}\right)$ & $k\left(\mathrm{~J} /\left(\mathrm{m} \cdot{ }^{\circ} \mathrm{C}\right)\right)$ & $c_{v}\left(\mathrm{~J} /\left(\mathrm{kg} \cdot{ }^{\circ} \mathrm{C}\right)\right)$ & $\alpha\left(1 /{ }^{\circ} \mathrm{C}\right)$ & $K_{\mathrm{IC}}\left(\mathrm{MPa} \cdot \mathrm{m}^{1 / 2}\right)$ \\
\hline $\mathrm{Al}$ & 71 & $1 / 3$ & 2700 & 18.1 & 880 & $8.5 e-6$ & 60 \\
Air & - & - & 1.204 & 0.02625 & 1.005 & - & - \\
\hline
\end{tabular}

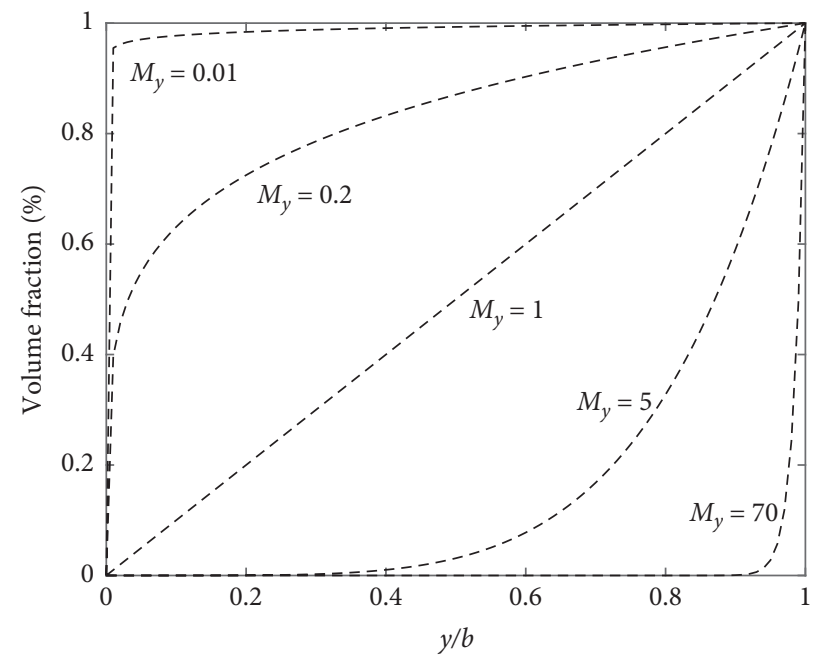

Figure 12: Volume fraction of metal.

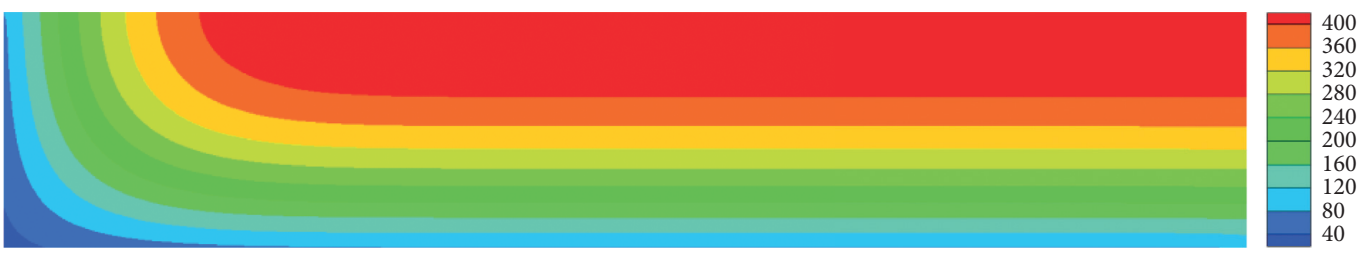

(a)

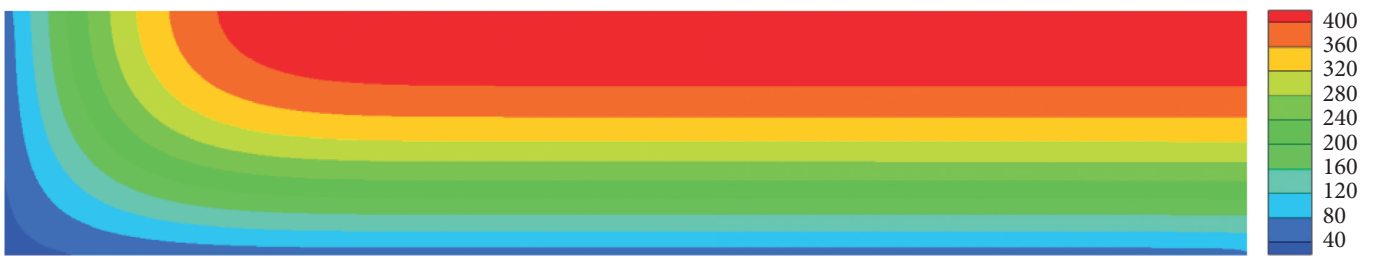

(b)

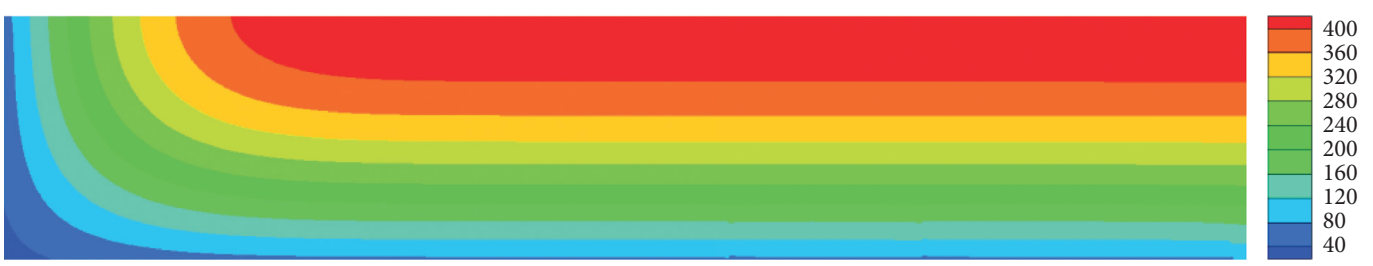

(c)

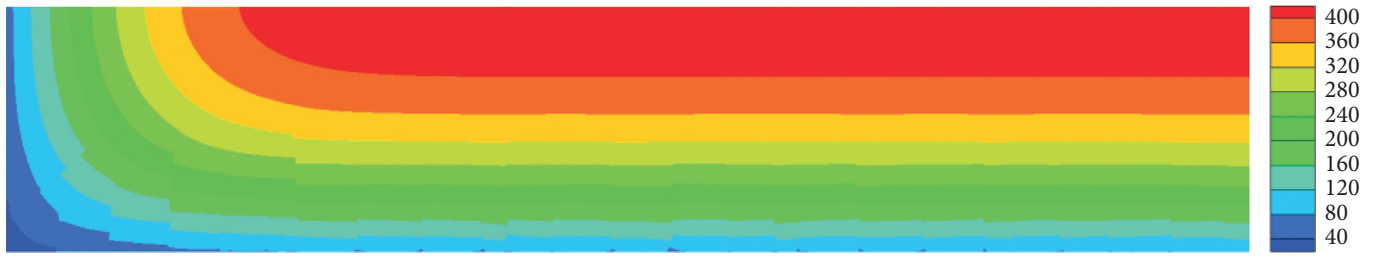

(d)

Figure 13: Continued. 


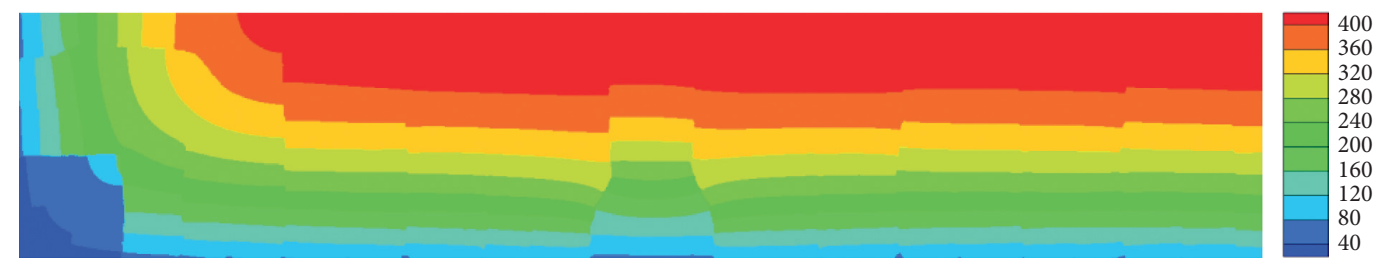

(e)

Figure 13: Temperature fields with different material shapes $\left(t=1.0 \mathrm{~s}\right.$ ): (a) $M_{y}=0.01$; (b) $M_{y}=0.2$; (c) $M_{y}=1$; (d) $M_{y}=5$; (e) $M_{y}=70$.

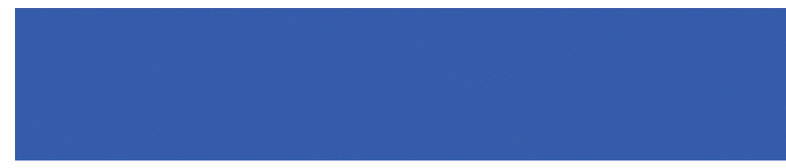

(a)

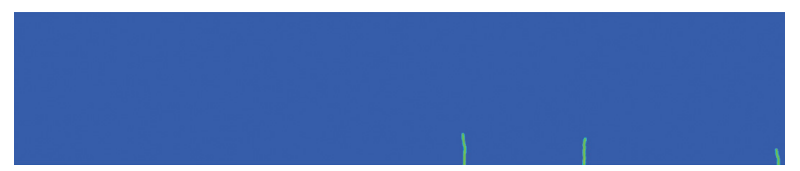

(c)

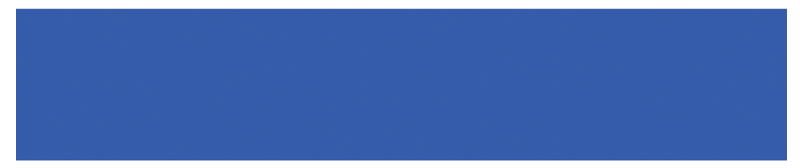

(b)

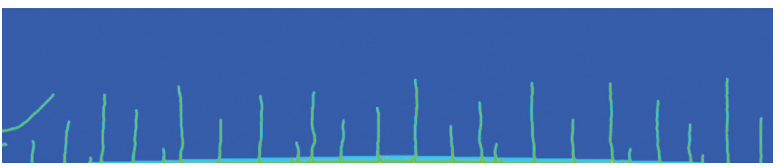

(d)

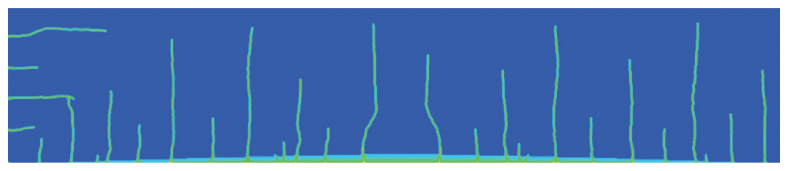

(e)

FiguRE 14: Thermal crack paths with different material shapes $\left(t=1.0 \mathrm{~s}\right.$ ): (a) $M_{y}=0.01$; (b) $M_{y}=0.2$; (c) $M_{y}=1$; (d) $M_{y}=5$; (e) $M_{y}=70$.

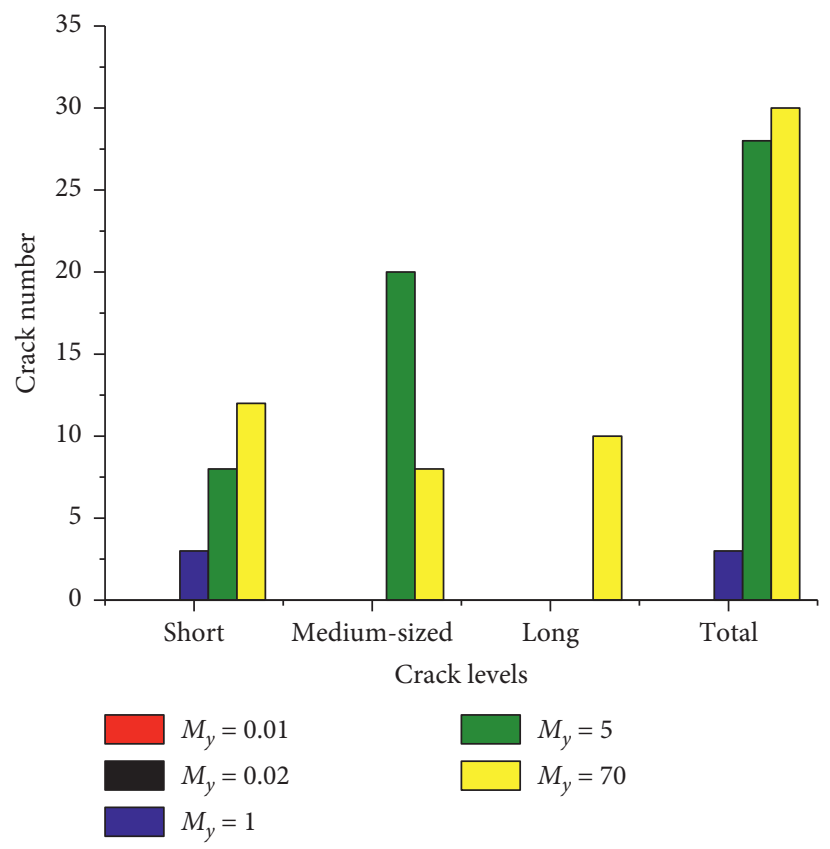

FIGURE 15: Thermal crack numbers with different material shapes $(t=1.0 \mathrm{~s})$. 
TABle 4: Average crack lengths with different material shapes $(t=1.0 \mathrm{~s})$.

\begin{tabular}{lccccc}
\hline$M_{y}$ & 0.01 & 0.2 & 1 & 5 & 70 \\
\hline $\bar{a}\left(a / L_{2}\right)$ & 0 & 0 & 0.186 & 0.336 & 0.449 \\
\hline
\end{tabular}

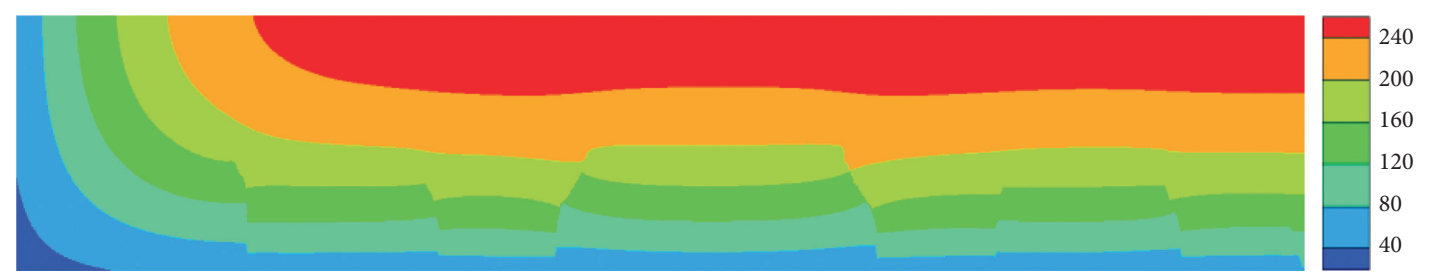

(a)

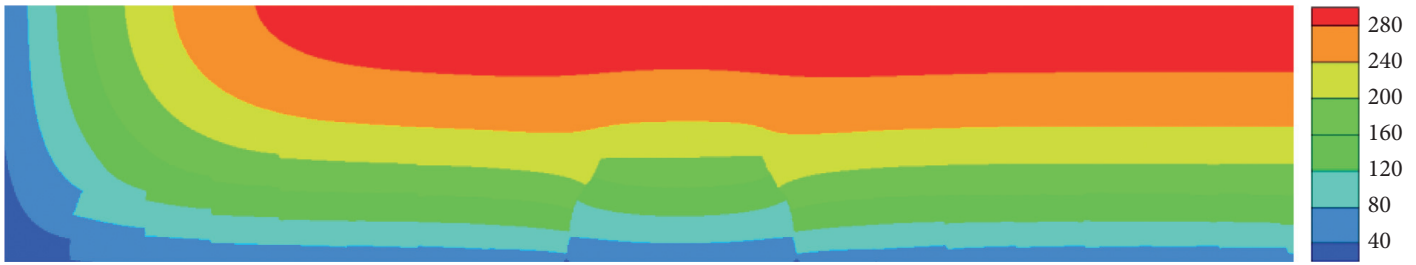

(b)

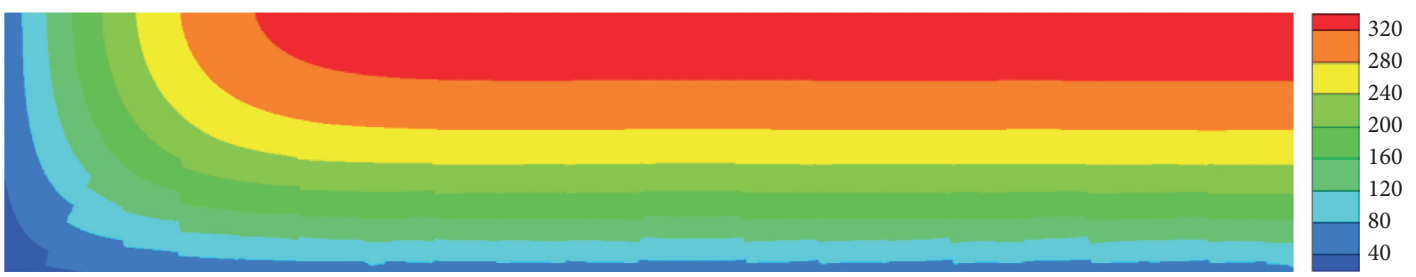

(c)

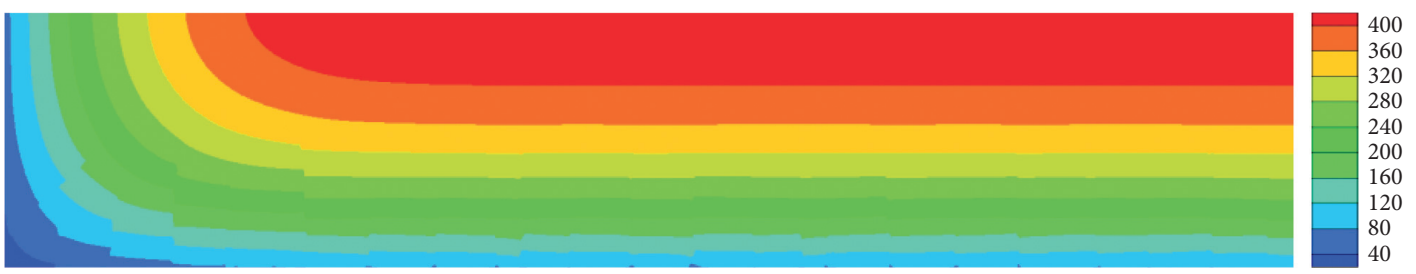

(d)

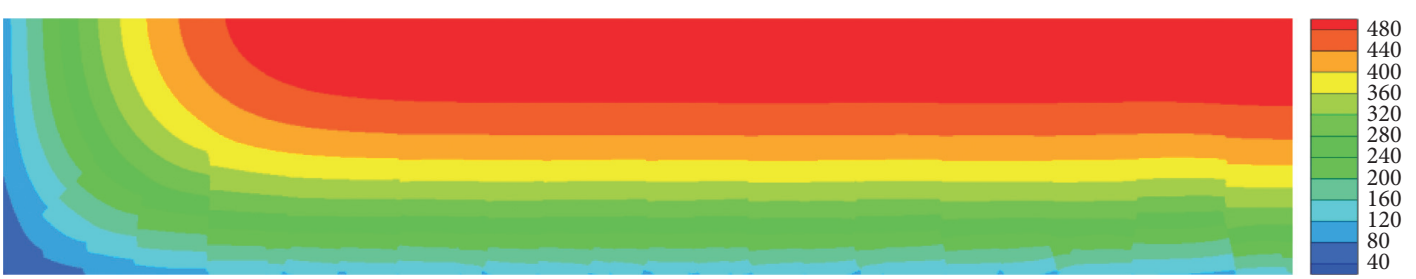

(e)

Figure 16: Temperature fields with different initial temperatures $(t=1.0 \mathrm{~s})$ : (a) $T_{0}=300^{\circ} \mathrm{C}$; (b) $T_{0}=350^{\circ} \mathrm{C}$; (c) $T_{0}=400^{\circ} \mathrm{C}$; (d) $T_{0}=500^{\circ} \mathrm{C}$; (e) $T_{0}=600^{\circ} \mathrm{C}$.

Environment temperature is $20^{\circ} \mathrm{C}$, and $M_{y}=5$. The predicted results at $t=1 \mathrm{~s}$ are shown in Figures 16 and 17.

In Figure 16, the frequency of temperature jump is enhanced when initial temperature is increasing. It can be found from Figures 17 and 18 that the numbers of short and total cracks tend to be increased due to the increasing initial temperature. The long crack is not emerged in the FGM slab, and the maximum crack length is about $0.5 L_{2}$. In addition, at the left-lower side of the FGM slab, thermal cracks are emerged when initial temperature increases. Associated with the results in Figure 14, the location of thermal crack initiated is also affected by the constrained conditions. 


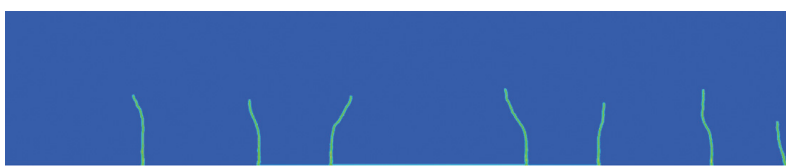

(a)

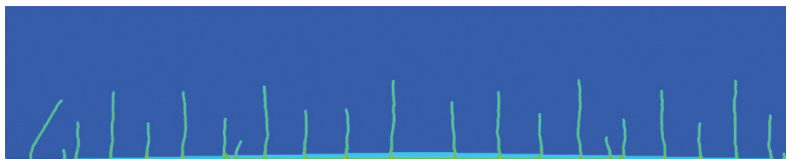

(c)

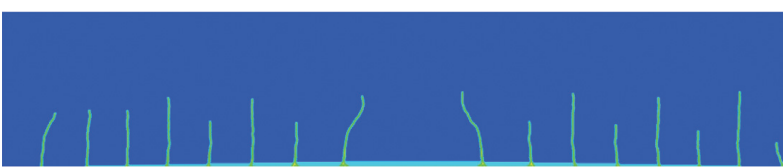

(b)

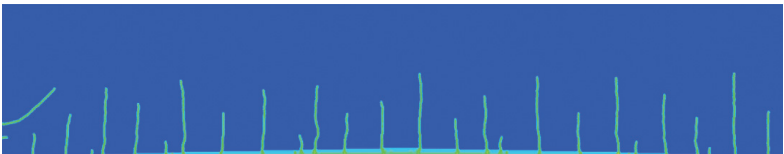

(d)

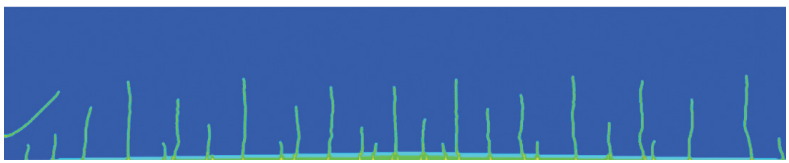

(e)

Figure 17: Thermal crack paths with different initial temperatures $(t=1.0 \mathrm{~s})$ : (a) $\mathrm{T}_{0}=300^{\circ} \mathrm{C}$; (b) $\mathrm{T}_{0}=350^{\circ} \mathrm{C}$; (c) $\mathrm{T}_{0}=400^{\circ} \mathrm{C}$; $(\mathrm{d}) \mathrm{T}_{0}=500^{\circ} \mathrm{C}$; (e) $\mathrm{T}_{0}=600^{\circ} \mathrm{C}$.

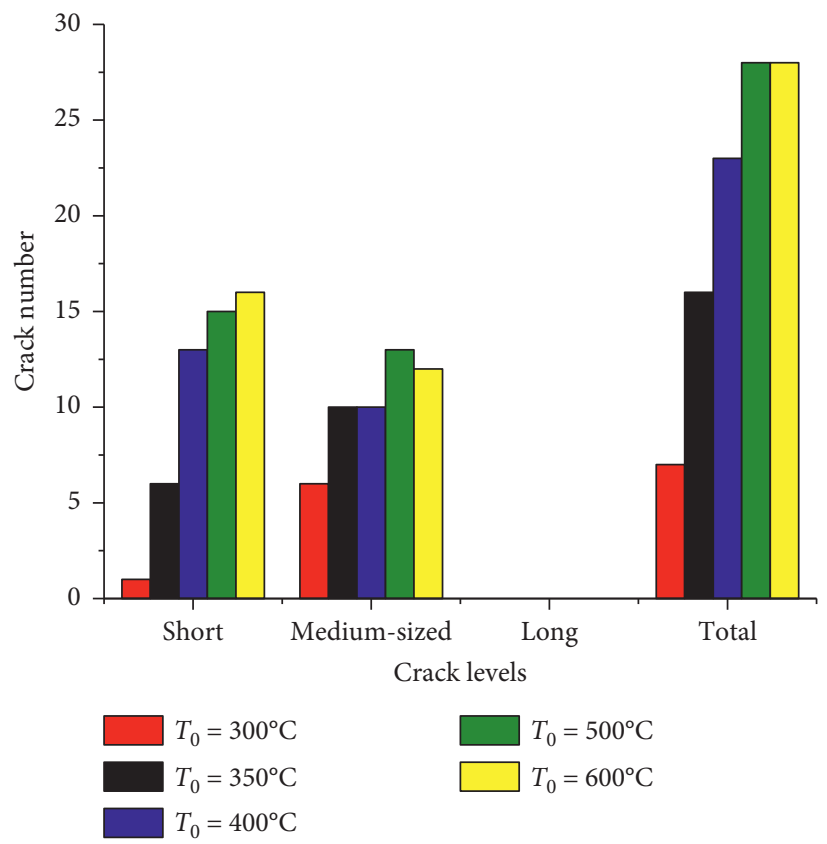

Figure 18: Thermal crack numbers with different initial temperatures $(t=1.0 \mathrm{~s})$.

TABle 5: Average crack lengths with different initial temperatures $(t=1.0 \mathrm{~s})$.

\begin{tabular}{lccccc}
\hline$T_{0}\left({ }^{\circ} \mathrm{C}\right)$ & 300 & 350 & 400 & 500 & 600 \\
\hline $\bar{a}\left(a / L_{2}\right)$ & 0.411 & 0.382 & 0.345 & 0.336 & 0.301 \\
\hline
\end{tabular}

The average crack lengths with different initial temperatures are given in Table 5. The average crack length is smaller when initial temperature is higher, owing to the increasing short crack number.

5.4. The Effect of Ceramic Fracture Toughness. The environment and the initial temperatures are $20^{\circ} \mathrm{C}$ and $500^{\circ} \mathrm{C}$, respectively, and $M_{y}=5$. The temperature fields and thermal crack paths with a different ceramic fracture toughness are calculated. The ceramic fracture toughness $K_{\mathrm{IC}-c}$ is specified as $2,3,4$, and $5 \mathrm{MPa} \cdot \mathrm{m}^{1 / 2}$. The results at $t=1 \mathrm{~s}$ are given in Figures 19 and 20.

The fracture toughness has no effect on heat conduction. The differences of temperature fields are caused by crack propagation (see Figure 19). Hence, the distinct temperature jumps are produced by a different ceramic 

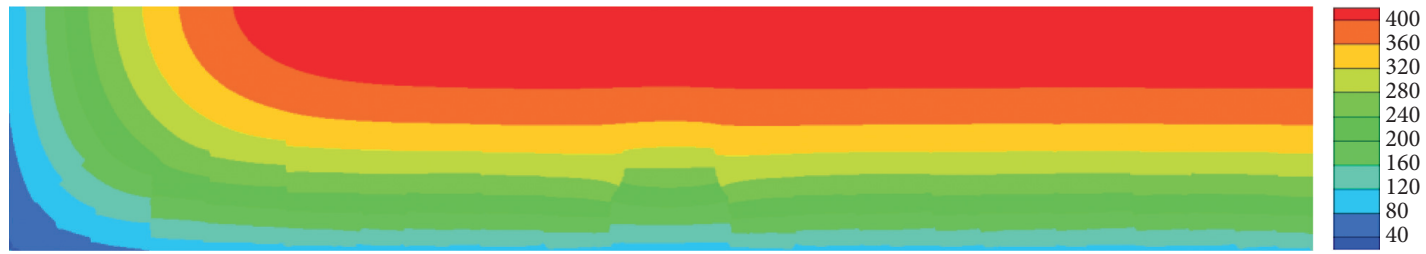

(a)
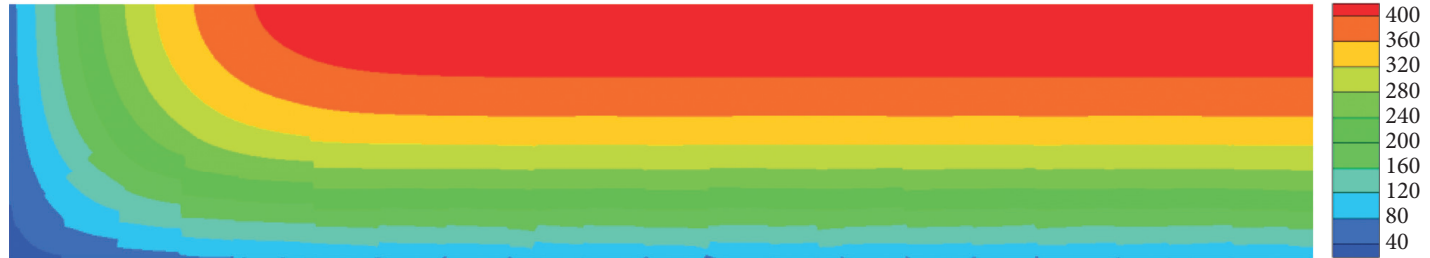

(b)
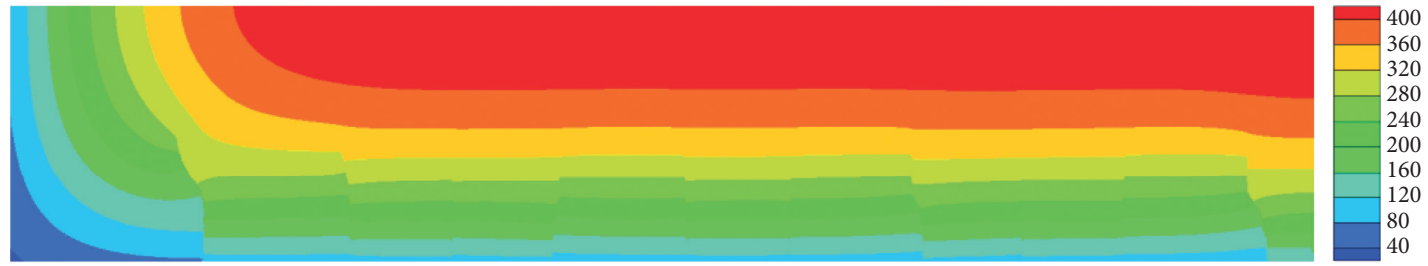

(c)
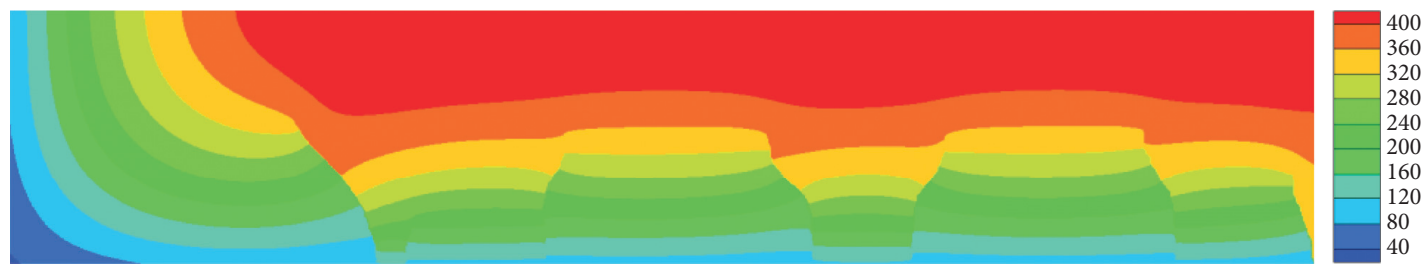

(d)

FIGURE 19: Temperature fields with a different ceramic fracture toughness $(t=1.0 \mathrm{~s}):(\mathrm{a}) K_{\mathrm{IC}-c}=2 \mathrm{MPa} \cdot \mathrm{m}^{1 / 2}$; (b) $K_{\mathrm{IC}-c}=3 \mathrm{MPa} \cdot \mathrm{m}^{1 / 2}$; (c) $K_{\mathrm{IC}-c}=4 \mathrm{MPa} \cdot \mathrm{m}^{1 / 2}$; (d) $K_{\mathrm{IC}-\mathrm{c}}=5 \mathrm{MPa} \cdot \mathrm{m}^{1 / 2}$.

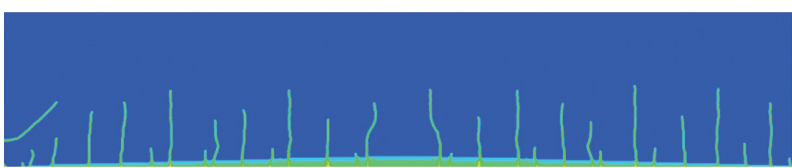

(a)

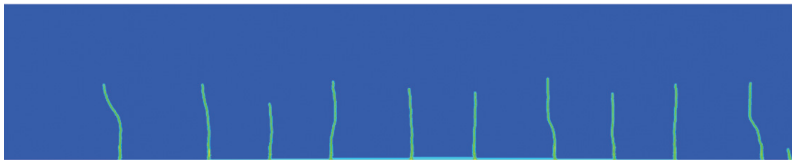

(c)

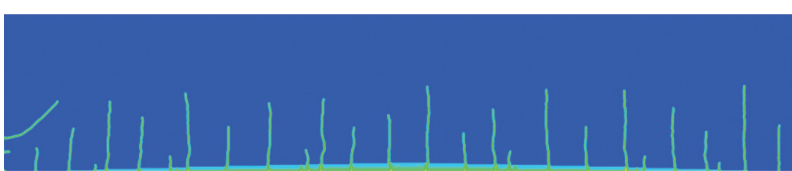

(b)

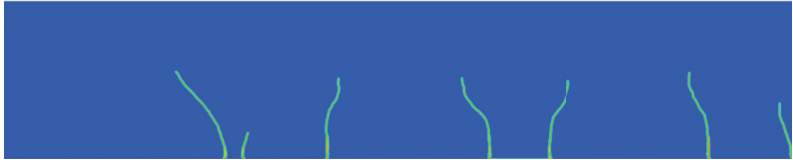

(d)

FIgURE 20: Thermal crack paths with a different ceramic fracture toughness $(t=1.0 \mathrm{~s}):(\mathrm{a}) K_{\mathrm{IC}-c}=2 \mathrm{MPa} \cdot \mathrm{m}^{1 / 2} ;(\mathrm{b}) K_{\mathrm{IC}-c}=3 \mathrm{MPa} \cdot \mathrm{m}^{1 / 2} ;(\mathrm{c})$ $K_{\mathrm{IC}-c}=4 \mathrm{MPa} \cdot \mathrm{m}^{1 / 2} ;(\mathrm{d}) K_{\mathrm{IC}-c}=5 \mathrm{MPa} \cdot \mathrm{m}^{1 / 2}$.

fracture toughness which determines the thermal crack patterns shown in Figure 20.

In Figures 20 and 21, the numbers of medium-sized and total cracks are decreased with the increasing ceramic fracture toughness. The cracks initiated at the left-lower side are reduced. In Table 6, the average crack length is increased as a result of short crack arresting. 


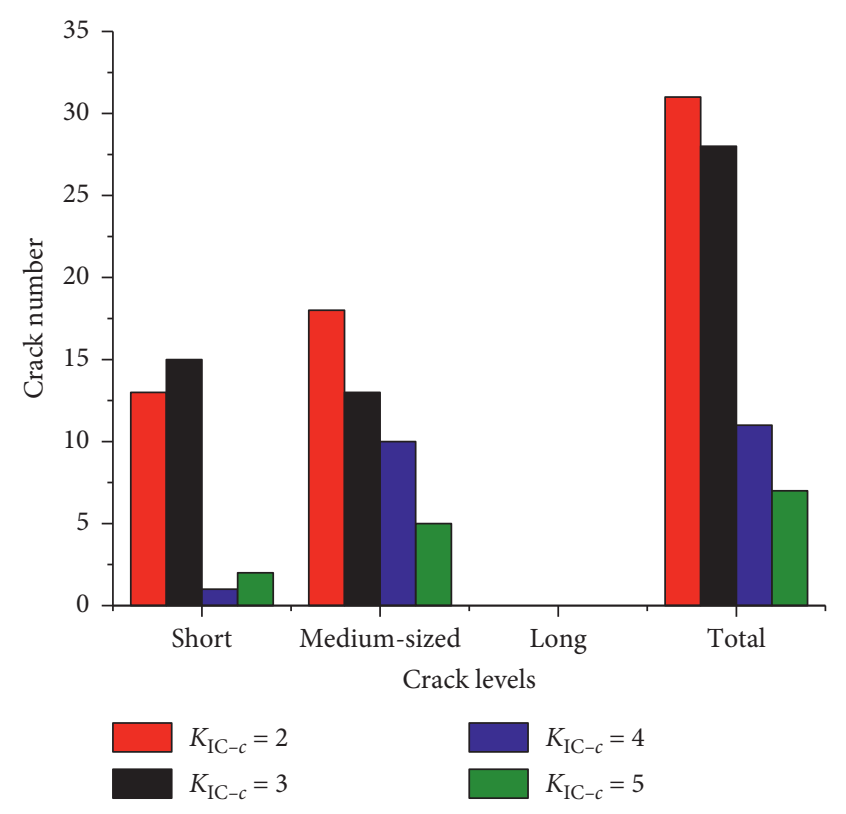

FIgURE 21: Thermal crack numbers with a different ceramic fracture toughness $(t=1.0 \mathrm{~s})$.

TABLE 6: Average crack lengths with a different ceramic fracture toughness $(t=1.0 \mathrm{~s})$.

\begin{tabular}{lcccc}
\hline$K_{\mathrm{IC}-c}\left(\mathrm{MPa} \cdot \mathrm{m}^{1 / 2}\right)$ & 2 & 3 & 4 & 5 \\
\hline $\bar{a}\left(a / L_{2}\right)$ & 0.303 & 0.336 & 0.421 & 0.430 \\
\hline
\end{tabular}

\section{Conclusions}

(1) A modified bond-based peridynamic thermomechanical model is developed to calculate thermal crack propagation. The crack numbers and paths of ceramic slab in quenching predicted by the modified PD model agree with the previous numerical and experimental ones. Compared with the original model, the calculation accuracy of the modified model for thermal cracking is improved.

(2) The thermal cracks in FGMs are still in periodical and hierarchical forms. The metal materials in FGMs can prevent crack initiation and arrest long cracks. The crack number and length are decreased when material shape parameter is increasing.

(3) The numbers of short and total cracks tend to be increased with the increasing initial temperature, while the strengthened ceramic fracture toughness can decrease the crack numbers.

\section{Data Availability}

The data used to support the findings of this study are available from the corresponding author upon request.

\section{Conflicts of Interest}

The authors declare that there are no conflicts of interest.

\section{Acknowledgments}

The authors are grateful for the financial support from the National Natural Science Foundation of China (Grant no. 91860128 and Grant no. 11572250).

\section{References}

[1] V. N. Burlayenko, H. Altenbach, T. Sadowski, and S. D. Dimitrova, "Computational simulations of thermal shock cracking by the virtual crack closure technique in functionally graded plate," Computational Materials Science, vol. 116, pp. 11-21, 2016.

[2] A. Gupta and M. Talha, "Recent development in modeling and analysis of functionally graded materials and structures," Progress in Aerospace Sciences, vol. 79, pp. 1-14, 2015.

[3] F. Bobaru, "Designing optimal volume fractions for functionally graded materials with temperature-dependent material properties," Journal of Applied Mechanics, vol. 74, no. 5, pp. 861-874, 2007.

[4] M. Kashtalyan and M. Menshykova, "Effect of a functionally graded interlayer on three-dimensional elastic deformation of coated plates subjected to transverse loading," Composite Structures, vol. 89, no. 2, pp. 167-176, 2009.

[5] H.-T. Thai and S.-E. Kim, "A review of theories for the modeling and analysis of functionally graded plates and shells," Composite Structures, vol. 128, pp. 70-86, 2015.

[6] G. Li, S. Guo, J. Zhang, Y. Li, and L. Han, "Transient heat conduction analysis of functionally graded materials by a multiple reciprocity boundary face method," Engineering Analysis with Boundary Elements, vol. 60, pp. 81-88, 2015.

[7] G.-Y. Huang, Y.-S. Wang, and S.-W. Yu, "A new model of functionally graded coatings with a crack under thermal loading," Journal of Thermal Stresses, vol. 27, no. 6, pp. 491-512, 2004.

[8] L. Guo and N. Noda, "Investigation methods for thermal shock crack problems of functionally graded materials-part I: analytical method," Journal of Thermal Stresses, vol. 37, no. 3 , pp. 292-324, 2014.

[9] N. Noda and Z.-H. Jin, "Stress singularity of nonhomogeneous body with crack in thermal stress fields," JSME International Journal. Ser. A, Mechanics and Material Engineering, vol. 38, no. 3, pp. 364-369, 1995.

[10] N. Noda and J. Zhi-He, "Thermal stress intensity factors for a crack in a strip of a functionally gradient material," International Journal of Solids and Structures, vol. 30, no. 8, pp. 1039-1056, 1993.

[11] N. Noda and B. L. Wang, "Transient thermoelastic responses of functionally graded materials containing collinear cracks," Engineering Fracture Mechanics, vol. 69, no. 14-16, pp. 1791-1809, 2002.

[12] V. Petrova and S. Schmauder, "Crack-interface crack interactions in functionally graded/homogeneous composite bimaterials subjected to a heat flux," Mechanics of Composite Materials, vol. 47, no. 1, pp. 125-136, 2011.

[13] V. Petrova and S. Schmauder, "Mathematical modelling and thermal stress intensity factors evaluation for an interface crack in the presence of a system of cracks in functionally graded/homogeneous bimaterials," Computational Materials Science, vol. 52, no. 1, pp. 171-177, 2012.

[14] L. Guo, N. Noda, and L. Wu, "Thermal fracture model for a functionally graded plate with a crack normal to the surfaces and arbitrary thermomechanical properties," Composite Science and Technology, vol. 68, no. 3-4, pp. 1034-1041, 2008. 
[15] T. Fujimoto and N. Noda, "Crack propagation in a functionally graded plate under thermal shock," Archive of Applied Mechanics (Ingenieur Archiv), vol. 70, no. 6, pp. 377-386, 2000.

[16] N. Noda, M. Ishihara, and N. Yamamoto, "Two-crack propagation paths in a functionally graded material plate subjected to thermal loadings," Journal of Thermal Stresses, vol. 27, no. 6, pp. 457-469, 2004.

[17] J.-H. Kim and G. H. Paulino, "Simulation of crack propagation in functionally graded materials under mixed-mode and nonproportional loading," International Journal of Mechanics and Materials in Design, vol. 1, no. 1, pp. 63-94, 2004.

[18] A. Oral, J. Lambros, and G. Anlas, "Crack initiation in functionally graded materials under mixed mode loading: experiments and simulations," Journal of Applied Mechanics, vol. 75, no. 5, Article ID 051110, 2008.

[19] S. S. Hosseini, H. Bayesteh, and S. Mohammadi, "Thermomechanical XFEM crack propagation analysis of functionally graded materials," Materials Science and Engineering: A, vol. 561, pp. 285-302, 2013.

[20] I. V. Ivanov, T. Sadowski, and D. Pietras, "Crack propagation in functionally graded strip under thermal shock," The European Physical Journal Special Topics, vol. 222, no. 7, pp. 1587-1595, 2013.

[21] H. Pathak, "Crack interaction study in functionally graded materials (FGMs) using XFEM under thermal and mechanical loading environment," Mechanics of Advanced Materials and Structures, pp. 1-24, 2018.

[22] G. Bhardwaj, I. V. Singh, B. K. Mishra, and T. Q. Bui, "Numerical simulation of functionally graded cracked plates using NURBS based XIGA under different loads and boundary conditions," Composite Structures, vol. 126, pp. 347-359, 2015.

[23] H. Pathak, "Three-dimensional quasi-static fatigue crack growth analysis in functionally graded materials (FGMs) using coupled FE-XEFG approach," Theoretical and Applied Fracture Mechanics, vol. 92, pp. 59-75, 2017.

[24] S. A. Silling, "Reformulation of elasticity theory for discontinuities and long-range forces," Journal of the Mechanics and Physics of Solids, vol. 48, no. 1, pp. 175-209, 2000.

[25] S. A. Silling and E. Askari, "A meshfree method based on the peridynamic model of solid mechanics," Computers and Structures, vol. 83, no. 17-18, pp. 1526-1535, 2005.

[26] B. Kilic and E. Madenci, "Prediction of crack paths in a quenched glass plate by using peridynamic theory," International Journal of Fracture, vol. 156, no. 2, pp. 165-177, 2009.

[27] A. Yuse and M. Sano, "Transition between crack patterns in quenched glass plate," Nature, vol. 362, no. 6418, pp. 329-331, 1993.

[28] P. D'Antuono and M. Morandini, "Thermal shock response via weakly coupled peridynamic thermo-mechanics," International Journal of Solids and Structures, vol. 129, pp. 74-89, 2017.

[29] S. Oterkus and E. Madenci, "Peridynamic modeling of fuel pellet cracking," Engineering Fracture Mechanics, vol. 176, pp. 23-37, 2017.

[30] H. Zhang and P. Qiao, "An extended state-based peridynamic model for damage growth prediction of bimaterial structures under thermomechanical loading," Engineering Fracture Mechanics, vol. 189, pp. 81-97, 2018.

[31] Y. Wang, X. Zhou, and M. Kou, "Peridynamic investigation on thermal fracturing behavior of ceramic nuclear fuel pellets under power cycles," Ceramics International, vol. 44, no. 10, pp. 11512-11542, 2018.

[32] Y. Wang, X. Zhou, and M. Kou, "Numerical studies on thermal shock crack branching instability in brittle solids," Engineering Fracture Mechanics, vol. 204, pp. 157-184, 2018.
[33] Y. Wang, X. Zhou, and M. Kou, “An improved coupled thermo-mechanic bond-based peridynamic model for cracking behaviors in brittle solids subjected to thermal shocks," European Journal of Mechanics-A/Solids, vol. 73, pp. 282-305, 2019.

[34] Y.-T. Wang and X.-P. Zhou, "Peridynamic simulation of thermal failure behaviors in rocks subjected to heating from boreholes," International Journal of Rock Mechanics and Mining Sciences, vol. 117, pp. 31-48, 2019.

[35] I. N. Giannakeas, T. K. Papathanasiou, and H. Bahai, "Simulation of thermal shock cracking in ceramics using bondbased peridynamics and FEM," Journal of the European Ceramic Society, vol. 38, no. 8, pp. 3037-3048, 2018.

[36] F. Bobaru and M. Duangpanya, "A peridynamic formulation for transient heat conduction in bodies with evolving discontinuities," Journal of Computational Physics, vol. 231, no. 7, pp. 2764-2785, 2012.

[37] A. Agwai, A peridynamic approach for coupled fields, Ph.D. thesis, University of Arizona, Tucson, AZ, USA, 2011.

[38] Z. Chen and F. Bobaru, "Selecting the kernel in a peridynamic formulation: a study for transient heat diffusion," Computer Physics Communications, vol. 197, pp. 51-60, 2015.

[39] Q. V. Le and F. Bobaru, "Surface corrections for peridynamic models in elasticity and fracture," Computational Mechanics, vol. 61, no. 4, pp. 499-518, 2018.

[40] E. Madenci and E. Oterkus, "Peridynamic theory and its applications," Springer, New York, NY, USA, 2014.

[41] B. Kilic, Peridynamic theory for progressive failure prediction in homogeneous and heterogeneous materials, Ph.D. thesis, University of Arizona, Tucson, AZ, USA, 2008.

[42] Z. Cheng, G. Zhang, Y. Wang, and F. Bobaru, "A peridynamic model for dynamic fracture in functionally graded materials," Composite Structures, vol. 133, pp. 529-546, 2015.

[43] B. Kilic and E. Madenci, "An adaptive dynamic relaxation method for quasi-static simulations suing the peridynamic theory," Theoretical and Applied Fracture Mechanics, vol. 53, no. 3, pp. 194-204, 2010.

[44] C. P. Jiang, X. F. Wu, J. Li et al., "A study of the mechanism of formation and numerical simulations of crack patterns in ceramics subjected to thermal shock," Acta Materialia, vol. 60, no. 11, pp. 4540-4550, 2012.

[45] J. Li, F. Song, and C. Jiang, "A non-local approach to crack process modeling in ceramic materials subjected to thermal shock," Engineering Fracture Mechanics, vol. 133, pp. 85-98, 2015.

[46] P. Sicsic, J.-J. Marigo, and C. Maurini, "Initiation of a periodic array of cracks in the thermal shock problem: a gradient damage modelling," Journal of the Mechanics and Physics of Solids, vol. 63, pp. 256-284, 2014.

[47] D. Chu, X. Li, and Z. Liu, "Study the dynamic crack path in brittle material under thermal shock loading by phase field modelling," International Journal of Fracture, vol. 208, no. 1-2, pp. 115-130, 2017.

[48] S. A. Silling, "Origin and effect of nonlocality in a composite," Journal of Mechanics of Materials and Structures, vol. 9, no. 2, pp. 245-258, 2014.

[49] J. Li, F. Song, and C. Jiang, "Direction numerical simulations on crack formation in ceramic materials under thermal shock by using a non-local fracture model," Journal of the European Ceramic Society, vol. 33, no. 13-14, pp. 2677-2687, 2013.

[50] S. B. Tang, H. Zhang, C. A. Tang, and H. Y. Liu, "Numerical model for the cracking behavior of heterogeneous brittle solids subject to thermal shock," International Journal of Solids and Structures, vol. 80, pp. 520-531, 2016. 